This item was submitted to Loughborough's Research Repository by the author.

Items in Figshare are protected by copyright, with all rights reserved, unless otherwise indicated.

\title{
An intelligent real-time cyber-physical toolset for energy and process prediction and optimisation in the future industrial Internet of Things
}

PLEASE CITE THE PUBLISHED VERSION

https://doi.org/10.1016/j.future.2017.09.026

\section{PUBLISHER}

(C) Elsevier

\section{VERSION}

AM (Accepted Manuscript)

\section{PUBLISHER STATEMENT}

This work is made available according to the conditions of the Creative Commons Attribution-NonCommercialNoDerivatives 4.0 International (CC BY-NC-ND 4.0) licence. Full details of this licence are available at: https://creativecommons.org/licenses/by-nc-nd/4.0/

\section{LICENCE}

CC BY-NC-ND 4.0

\section{REPOSITORY RECORD}

Pease, Sarogini, Russell Trueman, Callum Davies, Jude Grosberg, Kai Hin Yau, Navjot Kaur, Paul Conway, and Andrew West. 2017. "An Intelligent Real-time Cyber-physical Toolset for Energy and Process Prediction and Optimisation in the Future Industrial Internet of Things". Loughborough University. https://hdl.handle.net/2134/27124. 


\section{Accepted Manuscript}

An intelligent real-time cyber-physical toolset for energy and process

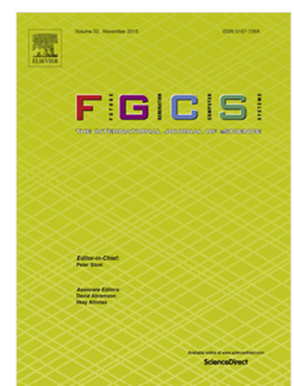
prediction and optimisation in the future industrial Internet of Things

Sarogini Grace Pease, Russell Trueman, Callum Davies, Jude Grosberg, Kai Hin Yau, Navjot Kaur, Paul Conway, Andrew West

PII:

S0167-739X(16)30382-X

DOI: $\quad$ https://doi.org/10.1016/j.future.2017.09.026

Reference: $\quad$ FUTURE 3681

To appear in: Future Generation Computer Systems

Received date : 6 October 2016

Revised date: 10 June 2017

Accepted date : 10 September 2017

Please cite this article as: S.G. Pease, R. Trueman, C. Davies, J. Grosberg, K.H. Yau, N. Kaur, P. Conway, A. West, An intelligent real-time cyber-physical toolset for energy and process prediction and optimisation in the future industrial Internet of Things, Future Generation Computer Systems (2017), https://doi.org/10.1016/j.future.2017.09.026

This is a PDF file of an unedited manuscript that has been accepted for publication. As a service to our customers we are providing this early version of the manuscript. The manuscript will undergo copyediting, typesetting, and review of the resulting proof before it is published in its final form. Please note that during the production process errors may be discovered which could affect the content, and all legal disclaimers that apply to the journal pertain. 


\title{
An Intelligent Real-time Cyber-Physical Toolset for Energy and Process Prediction and Optimisation in the Future Industrial Internet of Things
}

\author{
Sarogini Grace Pease, Russell Trueman, Callum Davies, Jude Grosberg, Kai Hin Yau, Navjot Kaur, Paul Conway, \\ and Andrew West \\ Wolfson School of Mechanical and Manufacturing Engineering, Loughborough University, Loughborough, Leicestershire, LE11 3TU UK
}

\begin{abstract}
Energy waste significantly contributes to increased costs in the automotive manufacturing industry, which is subject to energy usage restrictions and taxation from national and international policy makers and restrictions and charges from national energy providers. For example, the UK Climate Change Levy, charged to businesses at $0.554 \mathrm{p} / \mathrm{kWh}$ equates to $7.28 \%$ of a manufacturing business's energy bill based on an average total usage rate of $7.61 \mathrm{p} / \mathrm{kWh}$. Internet of Things (IoT) energy monitoring systems are being developed, however, there has been limited consideration of services for efficient energy-use and minimisation of production costs in industry. This paper presents the design, development and validation of a novel, adaptive Cyber-Physical Toolset to optimise cumulative plant energy consumption through characterisation and prediction of the active and reactive power of three-phase industrial machine processes. Extensive validation has been conducted in automotive manufacture production lines with industrial three-phase Hurco VM1 computer numerical control (CNC) machines.
\end{abstract}

Keywords:

\section{Introduction}

Consumption of energy resources on an industrial scale has a significant worldwide environmental impact, using around $54 \%$ of total energy production [1]. The automotive industry is subject to regulation and taxation from national and international policy makers [2] and restrictions and charges from national energy providers [3], thus a competitive economic advantage can be obtained by reducing unnecessary usage at a factory level [4]. For example, the UK Climate Change Levy (CCL) is charged to businesses at $0.554 \mathrm{p} / \mathrm{kWh}$. This equates to $7.28 \%$ of a manufacturing businesses energy bill based on an average total usage rate of $7.61 \mathrm{p} / \mathrm{kWh}$ [2]. The European Commission targets on climate change [5] mandate that member states increase energy efficiency by $20 \%$ by 2020 .

In the USA over $50 \%$ of energy produced is wasted, with energy-efficient technologies offering a $23 \%$ improvement (equivalent to 7\% GDP) [6]. UK waste equates to $£ 1$.6bn annually and could potentially be reduced by $15-30 \%$ [7, 8]. Appropriate optimisation first depends on characterising wasteful consumption with accurate monitoring of processes, associating these with their unique energy signatures [9].

Monitoring the resource consumption of each process is a key factor in achieving improved efficiency in industrial applications. Extraction of the features responsible for energy waste can be used to instruct intelligent process scheduling. In the domain of energy management, advantages can then be obtained through adaptation of industrial processes to reduce unnecessary waste and tuning process scheduling in line with energy pricing and excess charges.

Systems and assets in this domain must comply with industrial safety and robustness standards, e.g. wallmounting on DIN Rails and wiring enclosure [10]. Many proposed systems facilitate monitoring of low power $(<240 \mathrm{~V},<10 \mathrm{~A})$ domestic and small business systems [11]-[12]. In a typical manufacturing plant various machines, distributed in production lines, will operate in excess of the nominal three-phase voltage $(>400 \mathrm{~V})$ to perform a range of assembly and machining operations. Three-phase, high voltage ( $>1 k V_{R M S}$ [13]) Alternating Current (AC) supply systems are predominantly used to distribute electrical power and supply electricity directly around industrial sites in the UK. Three-phase, low voltage $\left(50 \mathrm{~V}-1 k V_{R M S}\right) \mathrm{AC}$ wiring 
will then distribute power to individual machines, in excess of $400 \mathrm{~V}$.

Energy in a three-phase AC system is distinguished into apparent, active and reactive energy. Active energy (measured in Watt hours) is part of the total energy consumption that is directly converted into heat (ohmic losses), motion or torque. Reactive energy (measured in VAR hours) is a function of capacitive and inductive loads such as the coil inductance of motors. Apparent energy (measured in VA hours) is a function of total impedance.

Active and reactive power are billed in non-domestic markets in line with the Distribution Connection and Use of System Agreement (DCUSA) [14], a contract between electricity suppliers, distributors and generators. Active power consumption is charged according to Distribution Use of System charges, a Maximum Import Capacity (MIC) limit of an industrial site, which is agreed on connection, and Maximum Demand (MD), the highest peak electricity flow during a half hour period per defined period (month or year). Additionally, half-hourly meters are charged under the Triad system, based on how much active power the 10,000 heaviest users consume during three undisclosed half-hour periods of peak demand between November and February $[15,16]$. Businesses are then significantly penalised for consumption at peak times [2].

Inductors and capacitors drop voltage and draw current, thus while Reactive power doesn't constitute a real load, this flow causes unwanted line losses and must be considered for the dimensioning of cables and transformers. Reactive power charges are applied by energy suppliers when reactive power use exceeds a predetermined percentage of total active power. Benchmarked energy monitoring approaches do not fully meet the challenges of characterising the unique active and reactive power profiles of automotive machining processes that non-domestic consumers are charged for $[17,18]$.

The Cyber-Physical Toolset is evaluated in the context of energy usage in the automotive industry. The majority of automotive engine block machining occurs on a dedicated transfer line where material is pulled through an ordered sequence of NC (G-code) operations on dedicated machining equipment (Section 4.1). Assuming a three-phase tariff of $0.25 \mathrm{p} / \mathrm{kWh}$ [19] and a daily consumption of 5 million $\mathrm{kWh}$ [20], reducing machining related waste by $10 \mathrm{p} / \mathrm{unit}$ could translate in to over $£ 50000$ in annual savings on one production line alone. Offering prediction and efficient use services enables industry to identify and avoid circumstances where levels are exceeded, prior to the end of a billing period. As an exam-

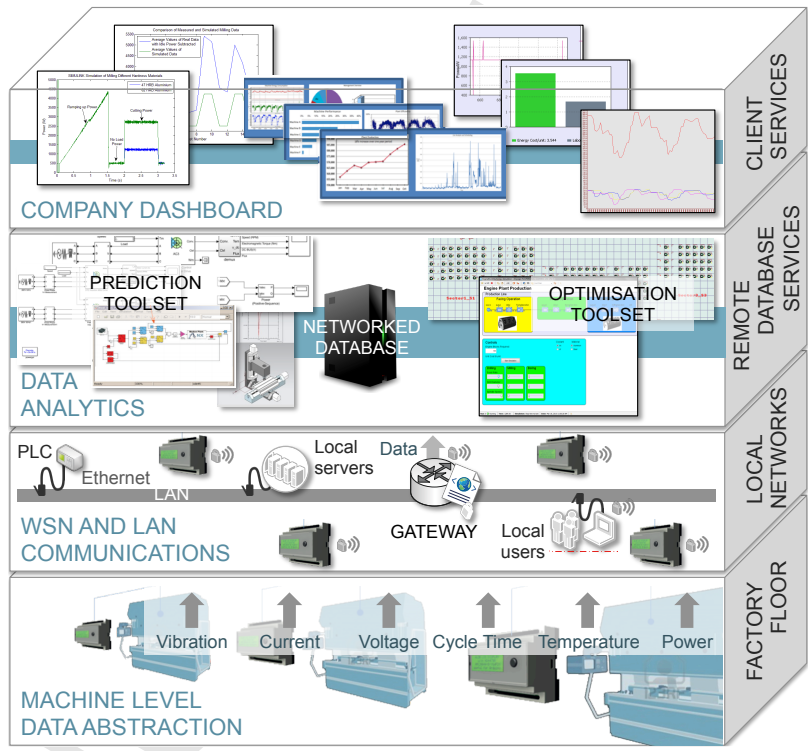

Figure 1: Four Layer Cyber-Physical Toolset

ple of the benefits these systems offer, Serbian car manufacturer Zastava reduced their energy consumption by over $25 \%$ through changes implemented as a result of energy monitoring [21].

Mass production puts greater emphasis on nonintrusive monitoring as pausing a production line to maintain or scale infrastructure represents high losses when, for example, one engine is produced every 30 seconds at the Ford factory in Dagenham [22].

Monitoring energy use and waste in industry can be supported by smart Internet of Things (IoT) systems. Low powered, low-cost Wireless Sensor Network (WSN) devices with long lifetimes can also meet industrial user-requirements of scalability and limited intrusion on supply chains and existing communications infrastructure [23, 24, 25].

Forming an Industrial IoT (IIoT) from these heterogeneous smart embedded devices attached to a global backbone, the Internet, then capitalises on the abstraction of knowledge and information by services and applications, for a market benefit [25]. Scalability and flexibility can also be provided with services for remote reconfiguration of energy monitoring parameters (e.g. communication frequency or voltage gain) and secure over-the-internet updates of embedded firmware. Financial advantages can be obtained through adaptation of industrial processes to reduce unnecessary energy waste and tuning process scheduling in line with energy pricing and excess charges. Scalability and flexibility can also be provided with services for remote reconfiguration of energy monitoring parameters (e.g. communication frequency or voltage gain) and secure over-the- 
Table 1: IIoT Energy Monitoring System Comparison

\begin{tabular}{|c|c|c|c|c|c|c|c|}
\hline \multirow{3}{*}{$\begin{array}{l}\text { System } \\
\text { Performance }\end{array}$} & \multicolumn{2}{|c|}{ Intrusiveness } & \multicolumn{2}{|c|}{ Scalability } & \multicolumn{3}{|c|}{ Industrial Application } \\
\hline & \multirow{2}{*}{$\begin{array}{l}\text { Low } \\
\text { Power }\end{array}$} & \multirow{2}{*}{$\begin{array}{l}\text { Network } \\
\text { Capability }\end{array}$} & \multirow{2}{*}{$\begin{array}{l}\text { Remote } \\
\text { Update }\end{array}$} & \multirow{2}{*}{$\begin{array}{l}\text { Comms } \\
\text { Freq. }\end{array}$} & \multicolumn{2}{|c|}{ Maximum } & \multirow{2}{*}{$\begin{array}{l}\text { Active and } \\
\text { Reactive Measurement }\end{array}$} \\
\hline & & & & & Current $[\mathrm{A}]$ & Voltage [V] & \\
\hline Cyber-physical Toolset & Yes & WSN/IoT & Yes & $200 \mathrm{~Hz}$ & 100 & 655 & Yes \\
\hline ACme $[45]$ & Yes & WSN/IoT & No & $\mathrm{n} / \mathrm{a}$ & 15 & 120 & Yes \\
\hline Plug [18] & No & WSN/IoT & No & $0.2 \mathrm{~Hz}$ & 8 & 280 & No \\
\hline Eco-eye [46] & Yes & WSN & No & $0.25 \mathrm{~Hz}$ & 200 & 255 & No \\
\hline Efergy [47] & Yes & WSN & No & $0.017 \mathrm{~Hz}$ & 95 & 300 & No \\
\hline \multicolumn{8}{|l|}{ OpenEnergyMonitor } \\
\hline +Emoncms [11] & Yes & None & No & $\mathrm{n} / \mathrm{a}$ & 97 & 240 & Yes \\
\hline iSensor [43] & Yes & None & No & $0.017 \mathrm{~Hz}$ & 10 & 240 & Yes \\
\hline O'Connell et al [44] & Yes & None & No & $1 \mathrm{~Hz}$ & 10 & 240 & Yes \\
\hline Siemens7KM [48] & No & Ethernet & No & $100 \mathrm{Mbps}$ & 150 & 720 & Yes \\
\hline Guimaraes et al [12] & Yes & Ethernet & No & $0.003 \mathrm{~Hz}$ & 10 & 220 & Yes \\
\hline
\end{tabular}

Table 2: System's Layers and Protocols

\begin{tabular}{ll}
\hline Layer & Protocol \\
\hline Application & IETF CoAP / REST Engine \\
Transport & UDP \\
Network & IPv6/RPL \\
Adaptation & 6LoWPAN \\
Duty Cycling & ContikiMAC \\
Physical & IEEE 802.15.4 \\
\hline
\end{tabular}

internet updates of embedded firmware.

A novel real-time power and process prediction and optimisation Cyber-Physical Toolset, that comprises four novel contributions, is proposed in this paper:

1. Novel embedded low power Dynamic Resource Monitors (DRM) and gateway devices, with comparable power monitoring to existing systems (Section 4), that are extended to one wireless system that directly monitors active and reactive loads drawing higher than nominal three-phase voltage $(>400 \mathrm{~V})$ and that meets industrial standards (Section 2.1). DRMs are incorporated into a scalable IoT architecture (Section 3.2) of WSN devices and remote servers, offering user access via databases and web interfaces, intelligent applications and client services (Section 2.2). Remote wireless reconfiguration services move away from intrusive adaptation offered by existing systems to system setting modification without interruption of machine or supply chain functioning (Section 3.3).
2. An energy and power consumption prediction service, used to generate a precise multi-body dynamics and discrete-event simulation visualisation of expected power usage on the basis of measurements of real automotive industry operations and processes (Section 2.3).

3. Initial processing and presentation of the large amounts of data output by energy monitoring by novel local and remote services for feature extraction (Section 3.1). An efficient control service then responds to time-varying energy pricing and productivity requirements to bound total plant energy consumption to variable billing thresholds, through identification of optimal in-plant scheduling of machine processes (Section 2.4).

4. Extensive validation of the prediction capabilities of the architecture against real measurements from an automotive production line with an industrial three-phase Hurco VM1 computer numerical control (CNC) machine. Demonstration of the capabilities to guarantee that total plant consumption and productivity remain within acceptable and guaranteed bounds, using intelligent process scheduling, to avoid peak charges and reduce waste (Sections 3.4-3.5).

\section{Cyber-physical Toolset}

The essential features of the Cyber-Physical Toolset are the remote and local, re-usable cyber-physical services for handling the big data output. Additionally, 


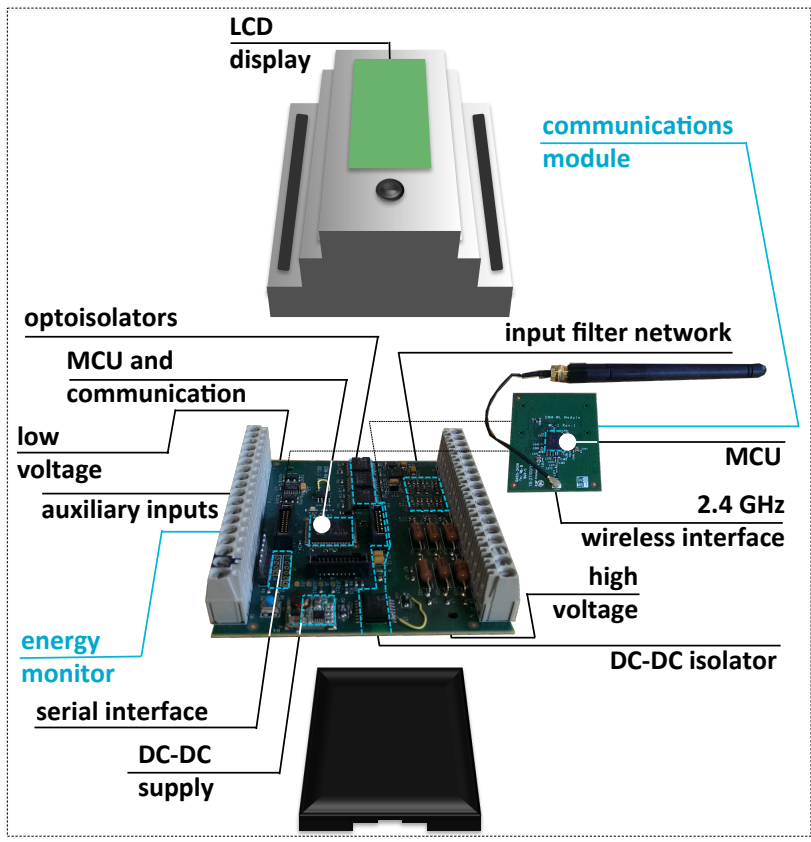

Figure 2: Component Features of DRM Devices

the devices and WSN protocols offer economy of infrastructure and low-power, robust scalability to heterogeneous machines and at the same time, timeliness and security of the wireless communications to ensure that these do not impact on data usefulness.

The DRM devices have been designed to meet the requirements of industrial energy monitoring: they combine low-power consumption with high-power (>400V) machine monitoring and are designed such that they can also be mounted on DIN Rails and in wiring enclosures [10] (Section 2.1).

The overall design of the energy monitoring architecture is divided into four layers: (1) factory floor direct power and energy monitoring with embedded devices, (2) WSN and LAN infrastructure, (3) data servers (database, back-up, data analysis for real-time actuation) and (4) applications and services, as shown in Figure 1.

All these devices are connected to the Internet and addressed with a unique IPv6 address, data are stored and analysed in remote servers. Local servers provide robustness against connectivity failure as data will be stored and analysed until Internet connectivity is restored. A comparison with existing systems is further evaluated in Table 1 and Section 4.2.

\subsection{Industrial Dynamic Resource Monitor Layer}

At device level there are two key components, the energy metering device and the communication device.

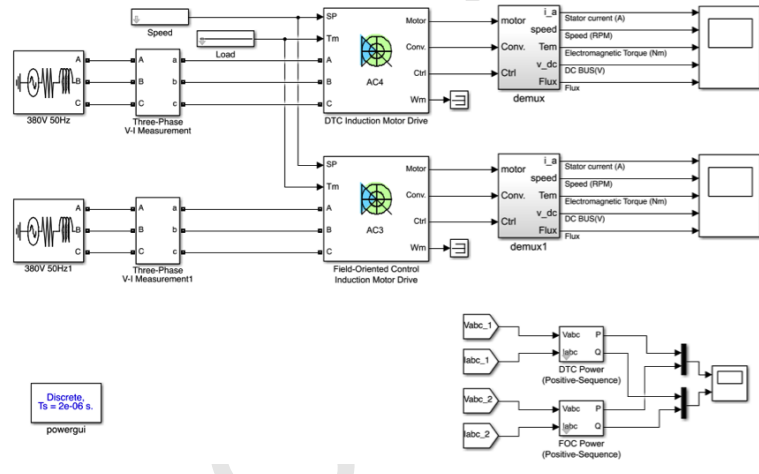

Figure 5: Prediction Service: DTC v. FOC Induction Motor Drives

The metering device is connected to a wireless communication device, based on the Texas Instruments (TI) CC2538+CC1200 devices [26]. Figure 2 illustrates the hardware used for energy and wireless communication, including sub-components. The devices operate with a $1.024 \mathrm{MHz}$ sampling rate and $40 \mathrm{~Hz}-2 \mathrm{KHz}$ report rate.

To ensure a high degree of accuracy and timing resolution, the DRMs use a dedicated ADE7878 IC meter for power calculation. This internally calculates real, reactive, and apparent power, active and reactive energy, to within $0.1 \%$ accuracy. High line voltages can be directly connected to the voltage input connections but are isolated from the low voltage side of the board (see Figure 2). This is because the line voltages are first attenuated using a resistor divider network, avoiding the need for voltage sensors e.g. voltage transducers.

Data are commuted to the microcontroller unit (MCU) over Serial Peripheral Interface (SPI) Bus. DRMs are equipped with a Cortex-M4 micro-controller that provides an additional Digital Signal Processing (DSP) unit and a Floating Point unit (FPU) [27]. These are essential to ensure the $168 \mathrm{MHz} \mathrm{MCU}$ can process data for real-time calculation of power signals and machine duty cycles. Devices can be directly connected to a Programmable Logic Controller (PLC) to support local actuation and configuration of machine state.

\subsection{IIoT Layers}

Table 2 details the protocols used in each conceptual layer by the DRM devices. The distributed approach to resource monitoring enables data transmission via any gateway device on the LAN to local and cloud-based services. Micro IPv6 (uIPv6) has been used, with its low memory requirements alongside UDP at the transport layer. In WSNs communication links are subject to nondeterministic layer- 1 and 2 dynamics [28] and occasionally links may become unreliable for periods of time due to interference. Thus, Routing for low Power and Lossy 


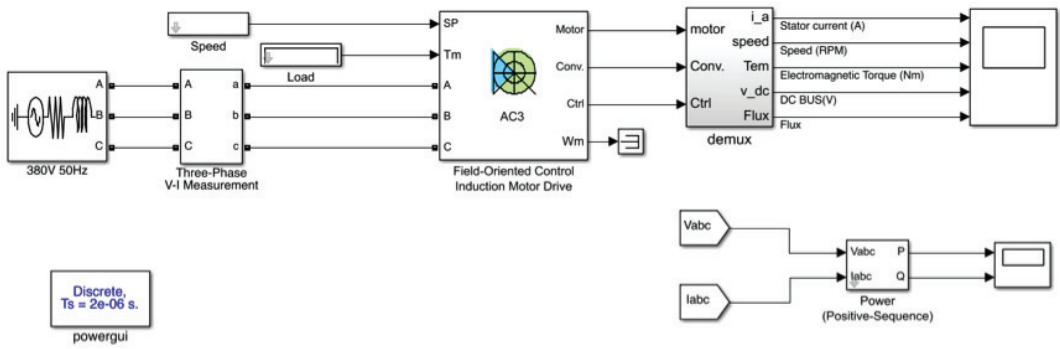

Figure 3: Prediction Service: Spindle Model

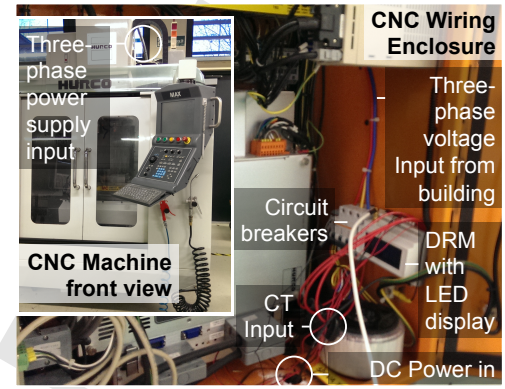

Figure 4: CNC Testbed with DRM networks (RPL) [29, 30] has been used as it is designed to not overreact to failures or attempt to stabilise under lossy wireless conditions. ContikiMAC [31] supports a range of radio duty cycles, allowing up to $99 \% \mathrm{MCU}$ sleep times, limiting device energy consumption.

Ethernet connected devices encrypt and transmit data over Transport Layer Security and Secure Sockets Layer (TLS/SSL) with HTTPS, using Restful services. Since wireless data can be easily sniffed, the Cyber-Physical Toolset uses hardware encryption and RSA public-key calculations to achieve data security.

To support web services, each application must adhere to a set of standardised protocols for sharing and accessing data. Thus they communicate, independent of operating system, database or programming language compatibility. Rules governing these interactions are pre-agreed. Few application protocols have been designed to meet the requirements of the IoT, such as energy and memory efficiency and cooperation with other building automation and machine-to-machine (M2M) applications.

The Constrained Application Protocol (CoAP) has been used as it is suitable for web services and applications over WSN. CoAP provides a REST-like interface [32] and adopts useful characteristics of HTTP such as resource abstraction, URI use, RESTful interaction, and extensible header options. At the same time, CoAP lowers bandwidth and implementationcomplexity overheads, in comparison to HTTP-based REST interfaces.

\subsection{Power Prediction Service}

The prediction service incorporates models developed in MATLAB Simulink. The service is used to generate a precise visualisation of expected power usage on the basis of particular machine operations and processes. These are then compared to measured active, reactive and apparent readings to tune and validate the simulations, which can predict usage in novel process scheduling scenarios. In this paper the component specifications and characteristics of a $\mathrm{CNC}$ machine are modelled.

The model comprises two motors, the axial and spindle motion, with an additional attached module, representing the structure and mass of components as constraints. These parameters have been taken from the specifications of a Hurco VM1 CNC machine [33]. The specifications used in the model and associated CAD model are given in Table 3. The spindle drive model is shown in Figure 3.

Due to the complexity of AC drives, the SimPowerSystems Library was used to implement a 7.5kW FieldOriented Control (FOC) Induction Motor Drive, connected to a $380 \mathrm{~V} 50 \mathrm{~Hz}$ three-phase voltage source. A Three-Phase V-I Measurement block was used to measure the three-phase currents and voltages, which were input into a Power (Positive-Sequence) block to compute the positive-sequence active and reactive powers. The speed reference and load torque were implemented as Stair Generator blocks, with the cutting torque calculated as $T_{c}=P_{c} / \omega$ where $\omega$ is the spindle speed in rads/s.

The model shown in Figure 5 was designed to compare the power consumption of two different types of AC drives: a Direct Torque Control (DTC) and a FieldOriented Control (FOC) drive. Each drive was connected to its own $380 \mathrm{~V} 50 \mathrm{~Hz}$ three-phase voltage source and controlled a $7.5 \mathrm{~kW}$ induction motor. As with the spindle model, the three-phase currents and voltages were measured using Three-Phase blocks, and input into Positive-Sequence blocks to again compute the active and reactive powers and power factor. The Power Factor is the ratio of power that produces work to power that generates inductive magnetic fields, it indicates the current drawn by a load for the amount of useful power transferred (see Section 4.3).

The induction motor model comprised an electrical 
Table 3: Input to Prediction Algorithm

\begin{tabular}{|c|c|c|}
\hline Component & Sub-System & Specification \\
\hline \multirow{3}{*}{ Spindle } & Motor & $\begin{array}{l}\text { AC Induction Motor, Single } \\
\text { winding, Fan cooled }\end{array}$ \\
\hline & Drive & $\begin{array}{l}200 \text { VAC, } 3 \text { Phase, } 50 / 60 \mathrm{~Hz} \text {, } \\
\text { Regenerative Braking }\end{array}$ \\
\hline & Power & $7.511 \mathrm{Kw}$ \\
\hline \multirow{3}{*}{ Movement and Ranges } & $\mathrm{X}$ & $660 \mathrm{~mm}$ \\
\hline & Y & $355.6 \mathrm{~mm}$ \\
\hline & $\mathrm{Z}$ & $457 \mathrm{~mm}$ \\
\hline Ball Screw Diameter & $\begin{array}{l}\mathrm{X}, \mathrm{Y} \\
\mathrm{Z}\end{array}$ & $\begin{array}{l}32 \mathrm{~mm} \\
40 \mathrm{~mm}\end{array}$ \\
\hline $\begin{array}{l}\text { Ball Screw Pitch } \\
\text { Coolant Pump Rating }\end{array}$ & $\mathrm{X}, \mathrm{Y}, \mathrm{Z}$ & $\begin{array}{l}12 \mathrm{~mm} \\
0.19 \mathrm{KW}\end{array}$ \\
\hline \multirow[t]{2}{*}{ Guide way Systems } & $\mathrm{X}, \mathrm{Y}$ & $\begin{array}{l}\text { Precision Linear Ball Rails, } \\
\text { 30mm Diameter }\end{array}$ \\
\hline & $\mathrm{Z}$ & $\begin{array}{l}\text { Precision Linear Ball Rails, } \\
\text { 35mm Diameter }\end{array}$ \\
\hline Axis Motor Torques & $\begin{array}{l}X, Y \\
Z\end{array}$ & $\begin{array}{l}5.39 / 13.8 \mathrm{Nm} \\
115 / 287 \mathrm{Nm}\end{array}$ \\
\hline
\end{tabular}

Table 4: Activities of AC Motor Model

\begin{tabular}{ll}
\hline Simulation Time (s) & Input \\
\hline 0 & Speed set point $=500 \mathrm{rpm}$ \\
0.5 & Load applied $(0,10,20,30,40 \mathrm{Nm})$ \\
1.5 & Load removed \\
1.75 & Speed set point $=0 \mathrm{rpm}$ \\
\hline
\end{tabular}

Table 5: Input to AC Motor

\begin{tabular}{lrrrrr}
\hline Load (Nm) & 0 & 10 & 20 & 30 & 40 \\
\hline FOC P (W) & 325.5 & 861.9 & 1432.0 & 2035.7 & 2672.6 \\
DTC P (W) & 326.7 & 863.6 & 1436.2 & 2044.3 & 2687.3 \\
Average (W) & 326.1 & 862.8 & 1434.1 & 2040.0 & 2680.0 \\
Percentage & & & & & \\
Difference & $0.37 \%$ & $0.20 \%$ & $0.29 \%$ & $0.42 \%$ & 0.55 \\
\hline
\end{tabular}

sub-model to output a current, a torque sub-model to output electromagnetic torque and a mechanical submodel to output rotor speed. Additionally, a stator current is required to calculate voltage drop across the supply cables in the voltage supply block. Simulation of an induction motor has been widely researched and in this paper the model presented in [61] is followed (see Section 4.4).

The speed reference and load torque have been implemented as Stair Generator blocks with the activities applied by the AC model during the course of the prediction simulation shown in Table 4. Figure 6 then demonstrates the predicted power profile for the $20 \mathrm{Nm}$ load case.

\subsection{Efficient Control Service}

The Lanner WITNESS discrete event simulator (WITNESS 14 Manufacturing Performance Edition) has been used to develop the Efficient Control service. WITNESS provides a comprehensive set of tools to model and simulate many business processes [64, 65].

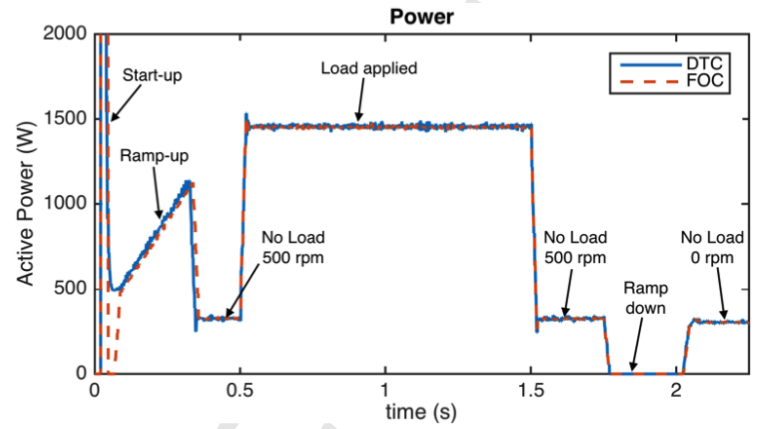

Figure 6: Power Profile with 20Nm Load

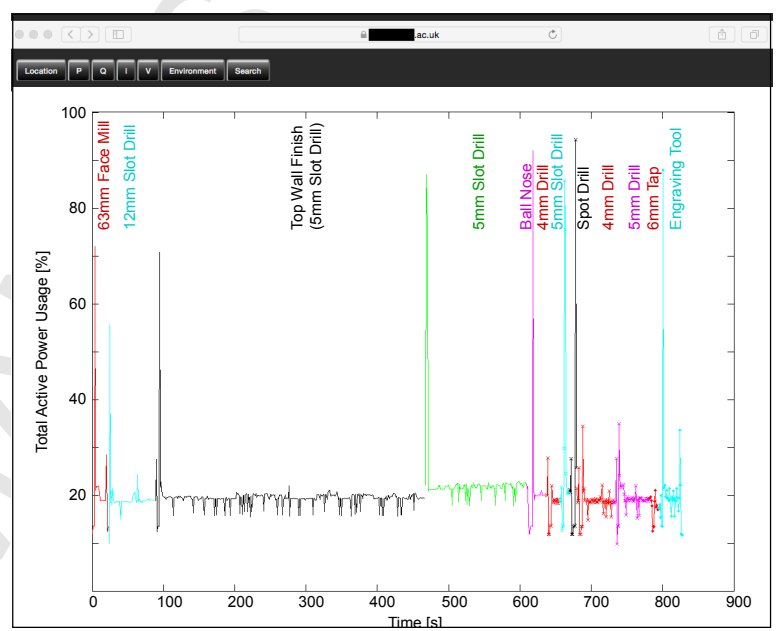

Figure 7: CNC Process Analysis of Active Power

A range of WITNESS elements (parts, people and machines $[64,65,66])$ have been combined with a set of logical rules to associate the process powers characterised in Section 3.1 with process control, sequencing and scheduling (see Section 3.5).

Functions import real process power values and output total power consumption and are combined with productivity and cost calculations to create a comprehensive factory model. The WITNESS built-in cost revenue and sustainability function [67] is used to define specific sustainability measures: energy in $\mathrm{kWh}$ and raw material consumed and productivity as batches of product generated.

Real-time measured energy consumption data is associated with characterised processes and used to populate the the Efficient Control service model of a production line. The service is then used to identify optimal in-plant scheduling of machine processes, in line with the KPIs of sustainable manufacturing evaluation [34]. The model enables total plant energy utilisation to be bounded within billing-defined limits and simultaneous prediction of cost/benefit and plant productivity. 
Localised changes to plant requirements can be used to tune the energy efficiency thresholds within the model. The key contribution of this model is that it is calibrated using energy signature data taken from realworld measurements of the energy profiles of the operations of a Hurco CNC machine, including milling, drilling, boring and facing (as detailed in Section 3.1).

Part elements are then used to represent the products on the manufacturing line and can be extended to represent product batches. Parts awaiting an operation are queued within buffer elements. These hold parts for a maximum period of time before they are pushed them to the next operation in the line or held for a period specified by the model to represent operations such as settling or cooling.

Machine elements are used to represent multiple $\mathrm{CNC}$ machines in a process queue. Within the service the operation time, time of and action on setup can all be modified prior to runtime. A sequence of working and non-working periods are represented using the WITNESS shift element and the built-in costing function is used to model the productivity of the system and to define when and how much energy each machine element will consume. The model will then output the number of operations performed, idle and busy times.

Results are provided to the user as the model is run, as a time-series graph of energy signatures over time. The model outputs both single phase and total power consumption curves and to record peak power consumption and signal to the user when this passes over a threshold value. Additionally, graphs of rate of change in power consumption, delay and throughput of part production are all provided by the service.

\section{Toolset Evaluation}

In this section we evaluate the Cyber-Physical Toolset in line with the key user-requirements in this research domain (Section 1):

- Industrial requirements: meeting safety and robustness standards [10]; functionality to monitor and limit typical manufacturing three-phase $\mathrm{AC}$ voltages $(>400 \mathrm{~V})$ and active and reactive power according to industrial MIC and MD limits (Sections 3.1 and 3.5).

- Scalability, adaptability and limited infrastructure intrusion: adding heterogeneous resources (e.g. software, hardware, virtualisation, data outputs and monitored machines) without disrupting a production line or reducing system performance (Sections 3.1,3.2 and 3.4).

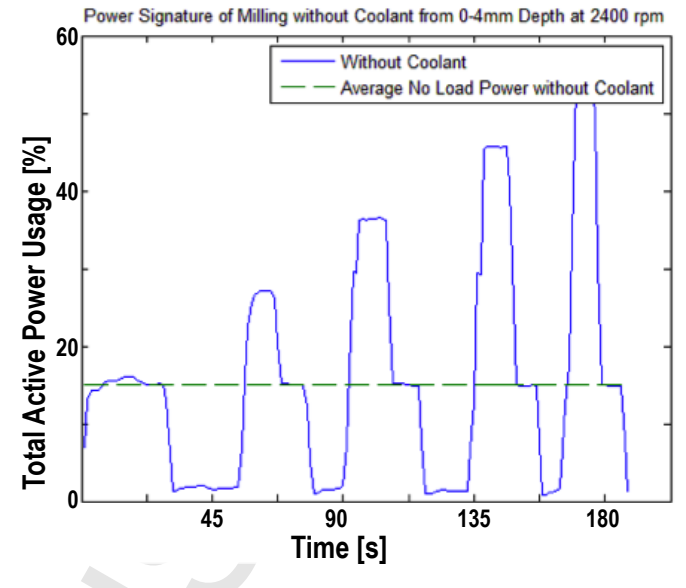

(a) Milling without Coolant

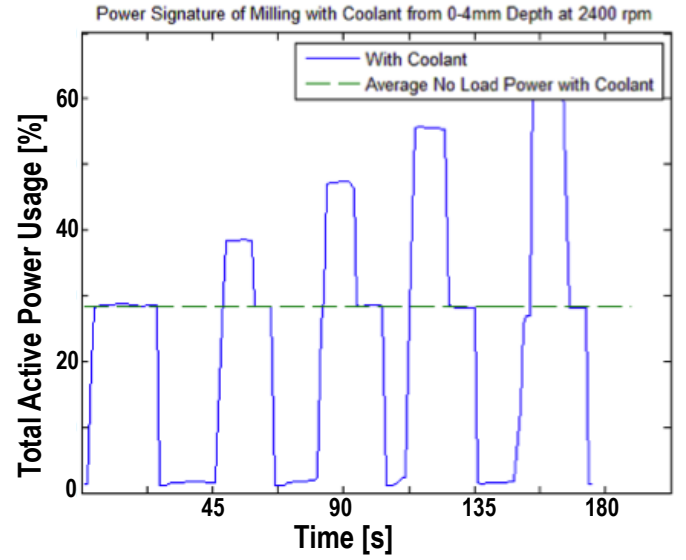

(b) Milling with Coolant

Figure 8: Total Active Power: 0-4mm Depth

- Contextualisation and flexibility: capability to decrease energy waste by contextualising energy data to manufacturing processes, with appropriate data granularity, timeliness and remote access and adaptation of systems to the knowledge abstracted, to support decision making (Sections 3.3 and 3.5).

The toolset has been validated to predict, monitor and control the power consumption of a Hurco VM1 $\mathrm{CNC}$ machine in an industrial environment for different processes, cutting parameters and work-piece materials. The embedded devices were mounted on DIN rails in the CNC wiring enclosure (see Figure 4). The $\mathrm{CNC}$ power usage was monitored when conducting slot drilling and milling of heat-treated aluminium alloys $\mathrm{Al}$ 6082 T6 and Al 6061 T6. Uncoated and TiN-coated car- 

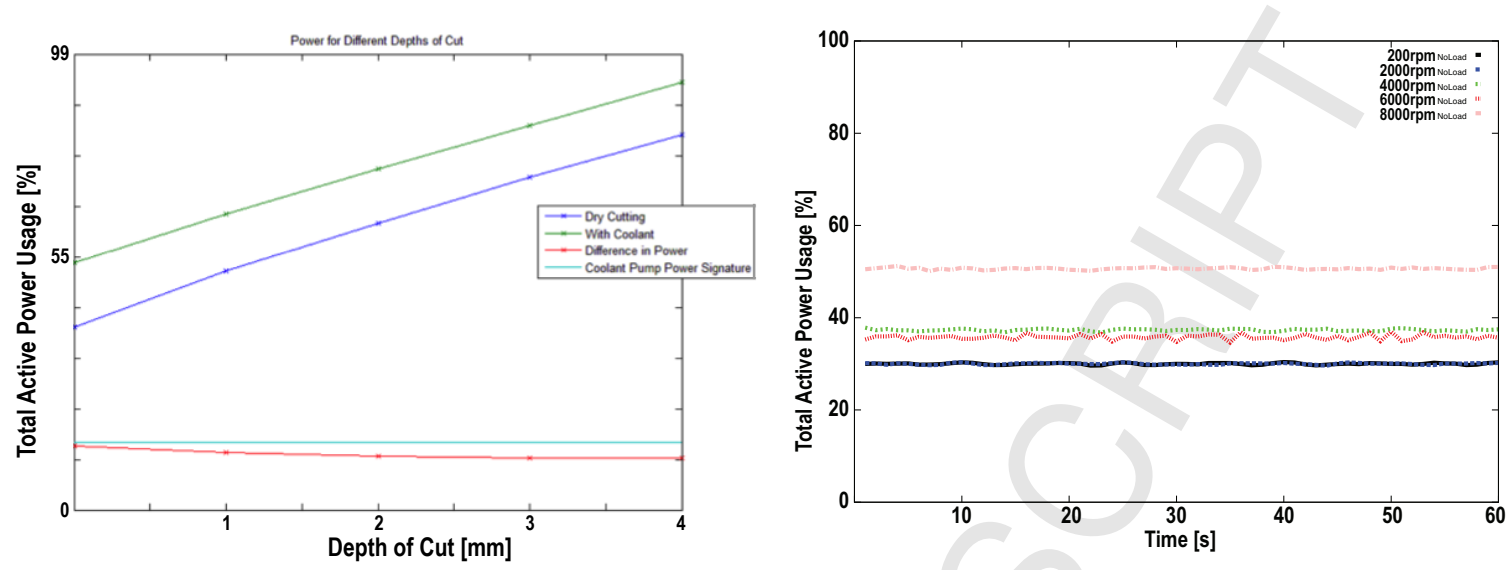

Figure 9: Total P with 0-4mm Depth of Cut (Mean)

Figure 10: Total P with Spindle Speed (Instantaneous)

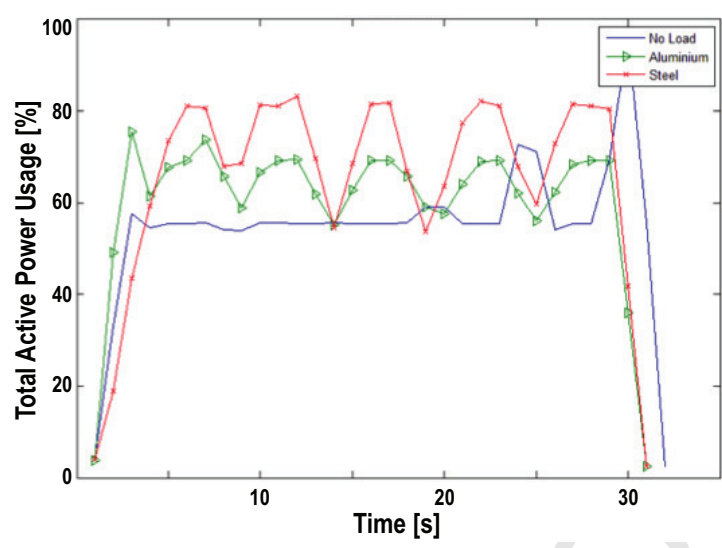

Figure 11: Total $\mathrm{P}$ for 2500rpm Drilling

bide tool inserts were used for a Kennametal $63 \mathrm{~mm}$ face Table 6: Depth of Cut Parameters

\begin{tabular}{ll}
\hline Parameter & Input \\
\hline Cutter diameter & $63 \mathrm{~mm}$ \\
Number of teeth & 5 \\
Approach angle & $45 \mathrm{deg}$ \\
Spindle speed & $2400 \mathrm{rpm}$ \\
Feed rate & $400 \mathrm{~mm} / \mathrm{min}$ \\
Width of cut & $25 \mathrm{~mm}$ (centred on work-piece) \\
Depth of cut & no load, 1, 2, 3, 4 mm \\
Material & Aluminium $(100 \mathrm{HB})$ \\
Coolant & On, Off \\
\hline
\end{tabular}

Table 7: HSS Twist Drill and Feed Rate Parameters

\begin{tabular}{ll}
\hline Parameter & Input \\
\hline Drill diameter & $8 \mathrm{~mm}$ \\
Feed rate & $120 \mathrm{~mm} / \mathrm{min}$ \\
Material & Aluminium $(100 \mathrm{HB})$, Steel $(179 \mathrm{HB})$ \\
Spindle speed & $2500,3000,3500 \mathrm{rpm}$ \\
\hline
\end{tabular}

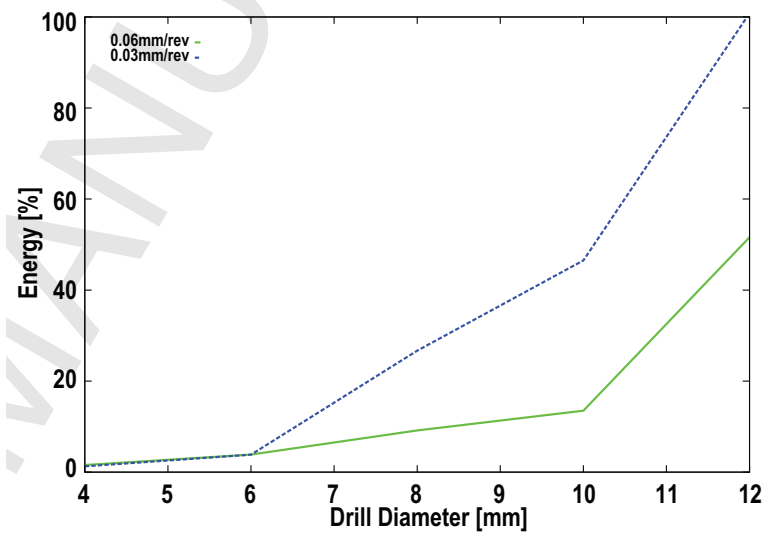

Figure 12: Energy Consumed with Feed Rate

mill. A ball nose cutter, taps and engraving tools were also used to produce a standard test piece.

\subsection{Characterising Machine Operations}

Process power usage was first analysed using the architecture to monitor a real Hurco VM1 CNC Machine in an industrial environment. Figure 7 indicates that through filtering and association of power consumption with process timing data, individual machine operations can be characterised to extract features on the usage of particular processes.

\subsubsection{Depth of Cut and Coolant}

Material removal, in isolation of contributing effects, was monitored in the next experiment. The only parameter changed was the depth of cut, thus any change in energy profile would be directly proportional to the depth of cut. It was possible to isolate the cutting energies involved in machining a component. The $63 \mathrm{~mm}$ 
face mill cutter was run through passes at depths of 1$4 \mathrm{~mm}$, repeated with and without coolant use. Assessment was made of the resulting surface finish according to coolant use. Table 6 details the tool; machine and material parameters used.

The milling energy profile displayed a characteristic peak energy during material removal and then a lower plateau during the no load period. The no load region in the dry cutting samples maintained a mean value of $1750.3 \mathrm{~W}$ (Figures 8[a]-[b]). The coolant pump consumed approximately $668 \mathrm{~W}$, this is comparable to the usage of the spindle motor, $671 \mathrm{~W}$ in the same experiment. Without coolant, a $10 \%$ reduction in power consumption can be a trade-off for potentially lower quality surface finish and increase in tool wear and breakage.

The maximum power usage for each depth of cut is compared in Figure 9. This demonstrates that the difference in power for dry cutting and cutting with coolant consistently matches the power signature of the coolant pump. This supports prediction of power required for a certain depth of cut by linear interpolation. A Taylor Hobson Talysurf stylus surface finish and contour measurement instrument was used to determine the surface texture parameter Mean Roughness ( $\mathrm{Ra}$ ) for the machined articles. A sampling length of $10 \mathrm{~mm}$ and a cut-off of $0.8 \mathrm{~mm}$ were used. Testing the surface finish indicated that the surface texture of the dry machined article was actually superior to that of the wet sample with Ra values of 0.0837 and 0.0878 respectfully.

Investigation into the usage of the spindle motor, operating with the same tool bit and not cutting any material is shown in Figure 10 for speeds of 200, 2000, 4000, 6000 and $8000 \mathrm{rpm}$. The power used to maintain a desired spindle speed generally increases nonlinearly with speed. Thus 20 and 2000rpm and 4000 and 6000rpm demonstrated similar power consumption values, with an increase up to $8000 \mathrm{rpm}$.

\subsubsection{Drilling}

Two experiments with drilling were performed: firstly, examining the influence of material properties on power consumption using a constant drill diameter and feed rate (Table 7) and secondly, the effect of drill diameter alone, utilising the same feed per revolution and cutting speed (Table 8).

Five holes were drilled into blocks of steel and aluminium and Figure 11 shows the no load, aluminium and steel energy profiles at 2500rpm. The five peaks indicate each drilled hole, requiring increased power for Steel over Aluminium due to the higher hardness and tensile strength of the material. Power usage dropped to the no-load level when no material was being removed.
Table 8: HSS Twist Drill Rate Parameters

\begin{tabular}{ll}
\hline Parameter & Input \\
\hline Cutting speed & $63 \mathrm{~m} / \mathrm{min}$ \\
Cutting feed & $0.03,0.06 \mathrm{~mm} / \mathrm{rev}$ \\
Material & Aluminium $(100 \mathrm{HB})$ \\
Drill diameter & $4,6,8,10,12 \mathrm{~mm}$ \\
\hline
\end{tabular}

Table 9: Performance for Aluminium and Steel Drilling

\begin{tabular}{lrrr}
\cline { 2 - 4 } Total Active Power & Aluminium & Steel & Difference \\
\hline Real Mean (W) & 880.04 & 1091.26 & 211.22 \\
Predicted Mean (W) & 685.10 & 980.51 & 295.42 \\
\cline { 2 - 4 } Error [\%] & 22.15 & 17.86 & - \\
\hline
\end{tabular}

Drill bit size was then varied between $4 \mathrm{~mm}$ and $12 \mathrm{~mm}$ in diameter, drilling five holes in a block of aluminium. Figure 12 demonstrates the energy consumed for different feed rates. Lower feed rates resulted in higher mean power consumption but a longer overall duration of the process, to a greater degree with increasing drill bit diameter. This could inform production line decisions relating to the balance between avoiding MIC charges due to high peak power and avoiding higher energy bills due to higher consumption.

\subsection{Infrastructure Evaluation}

In contrast to wired approaches discussed in Section 4.2, the economised infrastructure requirements of wireless communication can be at the expense of reduced timeliness and data usefulness. Adding more devices in range of each other increases competition for channel access and bandwidth as well as collision errors and recovery time.

The aim is to demonstrate that the system can provide a reasonable level of network performance while monitoring multiple devices and differing traffic rates distributed across a factory. As timeliness of the system impacts strongly on the usefulness of the data, endto-end packet delay has been used as the performance metric that is representative of the scalability trade-off.

Thus, 15 DRMs were deployed across a university building, the devices communicated with a single gateway device, from which monitored power and energy consumption information was sent to a remote database server.

End-to-end delay was evaluated for the network under low $(1 \mathrm{~Hz}$ communications update rate, 64B payload) and high traffic conditions (5Hz update rate, 64B payload), using round trip time (RTT) from Wireshark on a laptop connected to the LAN. The results demonstrated that in a large-scale deployment, using the Cyber-Physical Toolset (Section 2), a low RTT could 


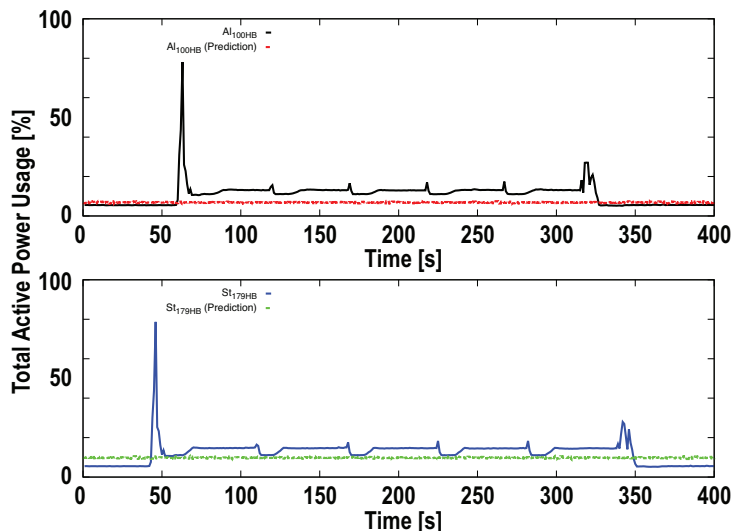

(a) Power Prediction: 2500rpm Drilling (Instantaneous)

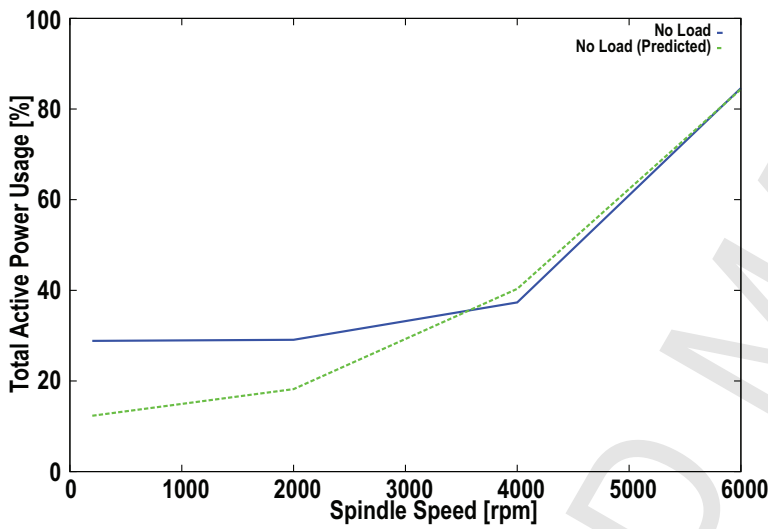

(c) Power Prediction: Spindle Speed (Mean)

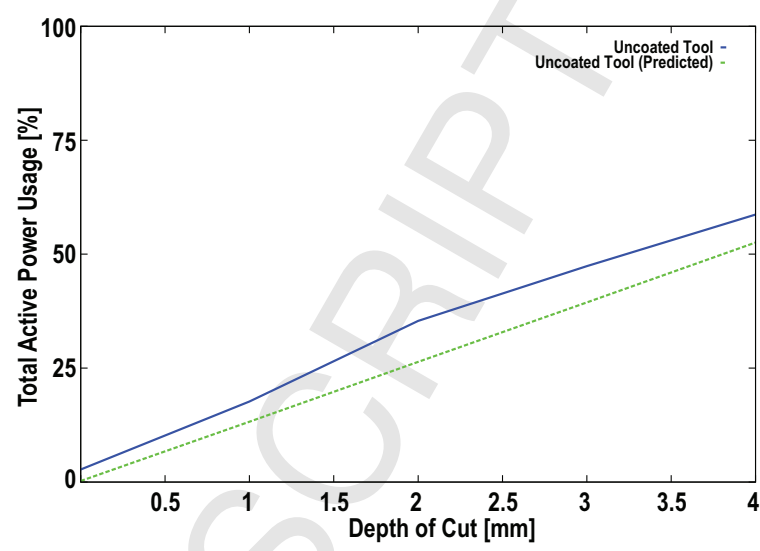

(b) Power Prediction: Milling Depth of Cut (Mean)

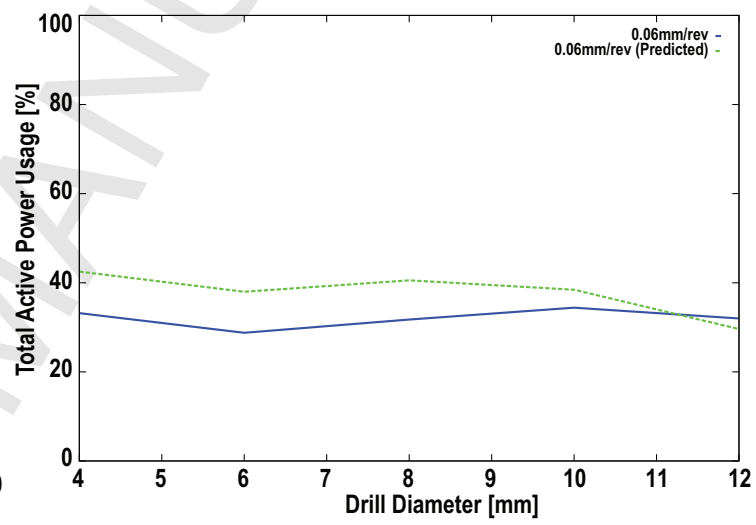

(d) Power Prediction: Drill Diameter (Mean)

Figure 13: Comparison of Predicted and Characterised Total Active Power

be maintained if the CCR, or wake-up configuration of ContikiMAC, was kept sufficiently high, at 1/32-1/64 $\mathrm{Hz}$ (Figure 14).

\subsection{Remote Reconfiguration of Communications}

Remote reconfiguration functionality has been designed for each gateway device to enable DRM parameters to be tuned by secure users. Gateway middleware monitors communications to abstract information on DRM's in range of the gateway. Users can then access a web interface to reconfigure a particular DRM, based on its unique ID.

This capability has been validated when the user wirelessly changes the update rate of the DRMs. Energy requirements vary across machines and processes and tuning output, in line with process frequencies,

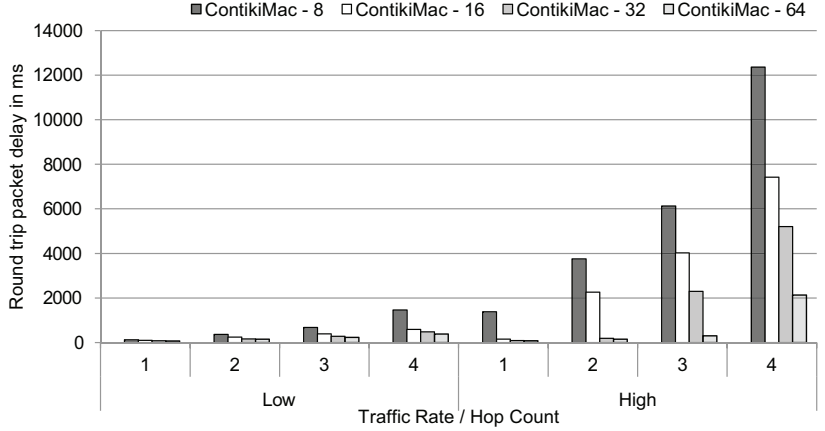

Figure 14: Performance with Varying Traffic and Wakeup Configurations

should be used to avoid unnecessary network congestion. While the DRMs sample at $1.024 \mathrm{MHz}$, given the limitations of the wireless medium, they can only up- 
date the database at up to 200 packets/second, though this is faster than other proposed systems (Table 1).

An initial experiment was conducted using an emulator testbed for a single-cycle energy profile, with a phase angle of zero input into the emulator. This signature enabled a validation of DRM output of Total Active Power, and of the capability to tune update frequency to meet feature extraction requirements.

The emulator testbed used LabVIEW to input energy profiles, to a National Instruments PXIe-1073 chassis with integrated controller. Two cards were installed in the emulator, one to measure the three-phase voltage input to the DRM from a real building power supply, and the other to produce a controllable emulation of three-
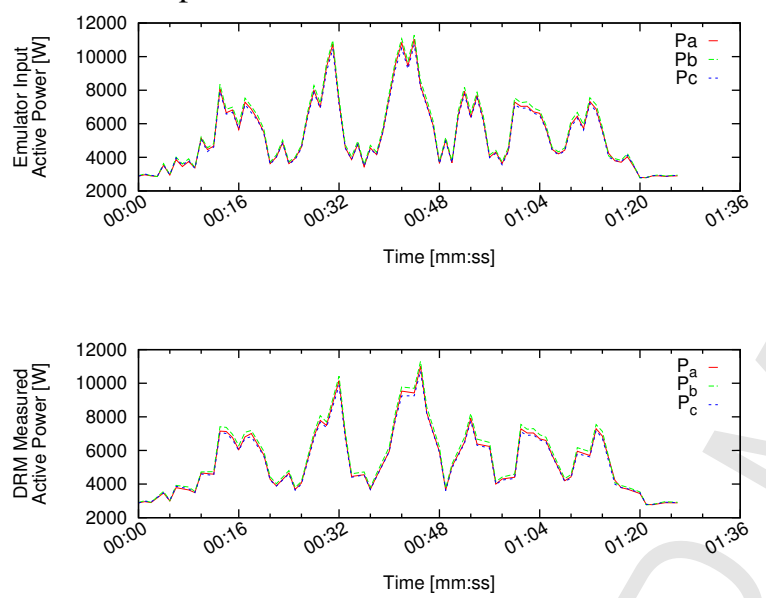

Figure 15: Input and DRM Output: Single Cycle, $1 \mathrm{~Hz}$ Update

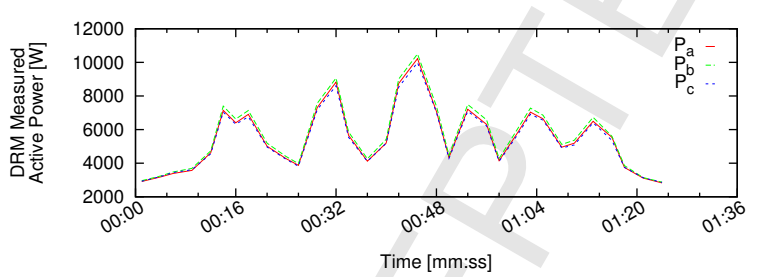

Figure 16: DRM Output: Single Cycle Active Power, $0.5 \mathrm{~Hz}$ Update

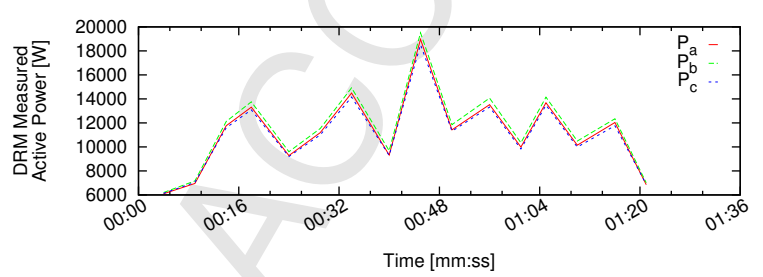

Figure 17: DRM Output: Single Cycle Active Power, $0.25 \mathrm{~Hz}$ Update phase current and deliver it to the DRM.

The currents and phase differences constructed in simulation were collected from real machine profiles for engine block post-machining operations, measured in the manufacturing line of a major automotive facility. The DRM unit was connected to the emulator via four wires for current input (one neutral) and provided with three voltage wire inputs from the main power supply. The building power supply lines form the three-phase voltage with a $120^{\circ}$ angle difference between line pairs. The LabVIEW diagram then adjusts the current amplitude and phase difference between current and line voltage, based on the input file.

The default update frequency of the DRMs is $1 \mathrm{~Hz}$ (1 packet/second), implemented to minimise communication overheads and corresponding energy consumption of the DRM. The mean of the data sampled by the DRM is transmitted for each update period. Use of the ADE7878 sampling rate of $1.024 \mathrm{MHz}$, report rate of $5000 \mathrm{~Hz}$ and variable update frequency supports accurate identification of cycle time and isolation of features within a cycle. Figure 15 illustrates a high level of accuracy of DRM measured Total Active Power, in comparison to this machine profile, input into the emulator.

Halving the frequency reduces the feature representation in the energy profile to larger operations and results in an apparent shortening of cycle time (Figure 16). With this dataset a $0.25 \mathrm{~Hz}$ update frequency then results in distortion, delay and temporal aliasing of the signal (Figure 17). This demonstrates that the capability to remotely tune update frequency can ensure a sufficiently high capability to reconstruct the input signal pattern when characterising the operations of a real machine.

\subsection{Power Prediction for Machining}

The prediction service output was compared to measured total active power consumption of the Hurco VM1 CNC in four experiments: (1) Comparison of drilling an Aluminium (100 Brinell hardness (HB)) and Steel (179HB) block with an $8 \mathrm{~mm}$ bit at 2500rpm (2) face milling at $0-4 \mathrm{~mm}$ depths of cut from an aluminium block, at $0.5 \mathrm{~mm}$ intervals (3) varying spindle speed from 0-6000rpm with no load, at 2000rpm intervals (4) Constant feed rate with varying drill bit diameters of 4$12 \mathrm{~mm}$. The service produces predicted power readings over time, however for comparison with the measured data over various datasets, mean active power has been used.

Figure 13[a] compares the predicted and real data for drilling aluminium and steel. The simulated values were conservative compared to the real values and were not capable of providing the precise characterisation of the 
process, including peaks surrounding component movement and spindle accelerations. In comparison to mean active power consumption they demonstrated a comparable trend for material drilled, as shown in Table 9. This shows that the predictions had a percentage error of between $17-23 \%$ and an RMS error of $16 \%$ overall.

Figure 13[b] demonstrates the active power for milling at various depths of cut from an aluminium block with a Kennametal $63 \mathrm{~mm}$ face mill. The service again provided a lower trend of estimated power consumption, with an RMS error of $10.5 \%$. The active power for varying spindle speed with no load are compared in Figure 13[c]. Without the impact of material feed rate on consumption, a higher accuracy was seen at higher spindle speeds. Although a similar non-linear trend was predicted in comparison to that measured.

In the next experiment, the cutting feed was kept at $0.06 \mathrm{~mm} / \mathrm{rev}$, by varying the spindle speed with changing drill bit diameters. As a result, the larger diameter drill bits are used at lower feed rates. The spindle speed is a dominant factor in power prediction, whereas the volume of material removed has a greater impact on power consumption in reality. Figure 13 [d] thus demonstrates that the accuracy of the estimation increased with decreasing feed rate.

\subsection{Efficient Control for Machining}

A warm-up period or starting condition was set in the Efficient Control service to create a non-empty starting state for the production line. The service includes timeshifts between five production lines to avoid simultaneous peaks in energy consumption. A delay function with different parameters is used to identify the production line and tune the start of an operation to achieve lower total plant consumption. The duty cycles of machines are also tuned to look at the effect on productivity of a line of different production plans.

The production lines, A to $\mathrm{E}$ and machines in each line were named numerically. Raw material supplied to the production line can be seen as RawMatA RawMatE that are pushed through machines numbered 001-009, representing pre-processing. The raw materials go to the queues where they are held until they can pass a Blockage element, used to prevent congestion as the materials pass subsequent processes. The MillingProc and DrillingProc elements represent feeding of raw materials into milling and drilling processes, respectively, with an associated energy signature (discussed in Section 3.1).

Five MillingProc in each production line were subsequent processes performed in a single machine, thus the Blockage element prevents the simultaneous arrival of

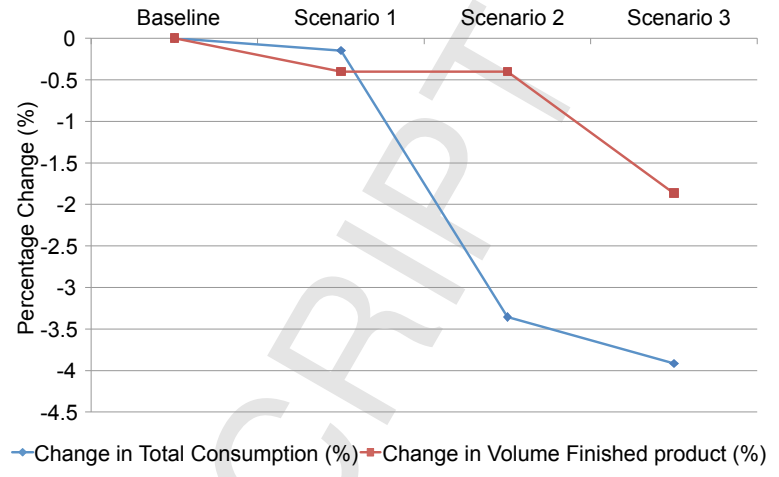

Figure 18: Efficient Production Line Control

raw material elements at a MillingProc. Raw materials are pushed to further queues to wait for the next process.

In this toolset, each DrillingProc represents five unique drilling processes with energy signatures for each. Raw materials are required to pass through 15 unique drilling processes, performed on a single machine, before leaving the production line.

The machine elements were set up as multicycle machines, taking as input the profiles of process energy signatures. A function was then defined to provide an ActiononFinish for each cycle, to add the instantaneous power usage to the total power consumption for the line. This allowed the full profile of an energy signature to be generated during each operation.

Power consumption is calculated separately by the service for each production line. The PowerA function calculates power consumption of production line A, as total and instantaneous power consumption, stored in the two variables TotalPowerA and CurrentPowerA.

TotalPowerA is obtained from the data collection elements, while instantaneous power is calculated as TotalPower $(n)$ - TotalPower $(n-1)$. A TotalPowerConsumption function is then used to calculate the total consumption of all lines, storing the calculated values in TotalReading.

TotalPowerConsumption is also used to calculate the peak consumption and record the sample count for the simulation period; these are stored in PeakConsumption and SampleCount. Finally, the TotalPowerConsumption function calculates the rate of change of power consumption, providing a dashboard graph for client access for each production line, plotted as the material is processed, enabling real-time monitoring of peak consumption.

The service has been designed to tune systems where raw material is supplied on demand, thus it does not include delays in material arrival, machine breakdown or production errors and the modelled plant is assumed 
to run 10 hours a day.

Validation was conducted for three optimisation algorithms against a baseline manufacturing scenario of 15 production lines:

Baseline: all machines operate at the same rate, without idle conditions or any energy reduction strategy.

Algorithm 1: raw material can only be pulled into subsequent processing stages at the first machine of both the MillingProc and DrillingProc if the total plant power consumption at that instant (TotalReading) is lower than a threshold capacity value (PlantCapacity set at $20000 \mathrm{~kW}$ ) and the rate of change of total power consumption (ChangeOfRate) is decreasing. Seven lines operated without restriction and eight with the logical constraint. Total consumption reduced by $0.14 \%$, with peak total consumption dropping to $82000 \mathrm{~kW}$ for a reduction of $0.41 \%$ in total finished product.

Algorithm 2: adds a second threshold to Algorithm 1 (LineCapacity set at $14000 \mathrm{~kW}$ ) defining the maximum capacity of a line at which raw material can be pulled into a production stage. Line operations can commence if line consumption is decreasing and below LineCapacity and if TotalReading is decreasing below PlantCapacity. With a reduced window within which machines could begin operations, total consumption reduced by $3.35 \%$ for a $0.40 \%$ drop in volume of finished product.

Algorithm 3: three production lines can commence operation when TotalReading is below a PlantCapacity of $20000 \mathrm{~kW}$ and line consumption is below LineCapacity and five lines only when TotalReading is decreasing and below a threshold Line Capacity of $14000 \mathrm{~kW}$. The remaining lines were unrestricted. Rapid increases in total sector consumption can lead to machine operations coming to a halt under this type of logical constraint. Peak total consumption was reduced by $3.91 \%$, however this was at the expense of a $1.87 \%$ reduction in volume of finished product.

Figure 18 demonstrates that using the second algorithm, the service is capable of controlling operations such that instantaneous total power consumption is bounded by a threshold (3.3\% of the cumulative load profile), with only a small decrease in the volume of total finished product $(0.4 \%)$. Limiting machines to only start an operation when total power con-

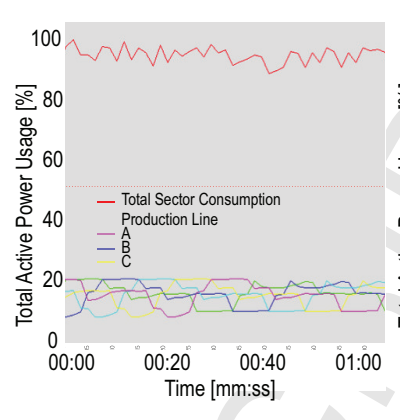

(a) Timing Offset

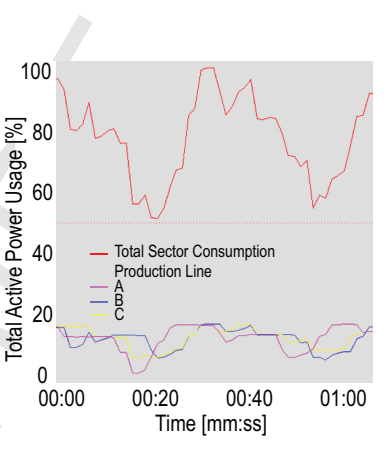

(b) Process Adjustment
Figure 19: Efficient Process Adjustment Test

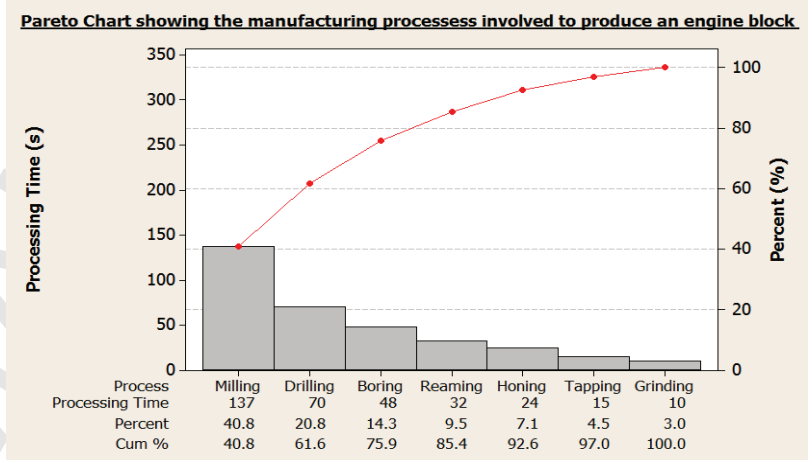

Figure 20: Machining Operations in Cylinder Block Manufacture

sumption is below the peak usage limit (arbitrarily set at PlantCapacity) can prevent individual production lines from pushing plant consumption into a region of higher billing or MIC excess charges. Permitting a process to start when TotalReading is decreasing avoids daisychaining of line start operations, which can push peak consumption beyond the threshold. Figure 19 shows that process adjustment outperforms a timing offset with line starts staggered.

\section{Related Work}

\subsection{Efficient Energy Usage}

In automotive manufacturing, engine blocks are predominantly produced through sand casting and lost foam casting. However, low dimensional accuracy and surface quality necessitate post-machining operations [35]. Of the machining process conducted on the transfer line, milling and drilling have been identified as key processes, taking over $60 \%$ of processing time in automotive Cylinder Block manufacture operations (see Figure $20[36,37])$. 
The cycle time of an engine block can be several minutes, and the Ford production line in Dagenham, Essex is able to produce upwards of 600000 diesel units per year on a single production line alone and over 1 million total units per year [38]. The two Ford manufacturing facilities within the UK consume approximately $300 \mathrm{GWh}$ of electricity annually [39] at a standard business rate tariff of $8 \mathrm{p} / \mathrm{kWh}[40,41]$, this translates to a $224 \mathrm{~m}$ UK energy bill excluding penalties, taxes and other charges.

\subsection{Power and Energy Monitoring}

OpenEnergyMonitor [11] is an open source threephase power metering system based on Arduino boards. Nodes measure apparent and real power, Root Mean Square (RMS) current and voltage, and transmit data over the Internet. With the addition of the Emoncms open source software platform, data can be accessed and visualised through a web application. This system has a $22 \mathrm{R}$ burden resistor which can measure up to 97A $(23.3 \mathrm{~kW}$ at $240 \mathrm{~V})$.

The iSensor used a combination of EpiSensor energy meters and ACme nodes to demonstrate real-time recognition and profiling of appliances using the RECAP appliance load monitoring system [42]. The system relies on full machine access and implementation begins with machine profiling and recognition phases to train an artificial neural network to monitor processes. Bauer et al [43] also investigated appliance recognition using power consumption. To achieve this, a deployment of ZigBee micro controllers combined with iSensor and 0-10A current transformer (CT)'s are used. However, both systems have been designed for monitoring singlephase consumer electronics.

O'Connell et al [44] used a REAM wireless actuation device, interfacing a CT to an energy metering integrated circuit (IC), for continuous socket sampling of current up to $10 \mathrm{~A}$ and voltage up to $240 \mathrm{~V}$. A PIC24 microcontroller was used for the calculation of RMS current and mains voltage, power factor and overall power. Actuation capabilities were demonstrated to reduce unnecessary heating by a $1.5 \mathrm{~kW}$ electric heater.

ACme [45] was developed as part of the Berkeley Wireless AC Meter/Switch project to provide active, reactive and apparent power measurement. It was one of the first IPv6 and 6LoWPAN implementations for remote energy measuring through WSN deployment. ACme uses the ADE7753 energy monitor chip for energy and power measurement, the Sharp solid-state relay for power switching, and the Berkeley Epic wireless module for communication. However, the system cannot support high power machine monitoring.
MIT's IoT Plug platform of sensor network devices for AC metering [18] employs power strips with sensing, networking, and computational capabilities. The platform provides apparent power measurements through a CT and uses an Analog-to-digital converter (ADC) for direct sampling. Power can be sensed and actuated at any of the four sockets on the strip. As with most sensor nodes, the Plug has a microcontroller and wireless transceiver but also contains embedded sensors including microphone and phototransistor. An expansion port allows the addition of extra sensors where required. However, the Plug has limited industrial application as it cannot measure active or reactive power or meet industrial mounting and enclosure requirements [10].

Existing higher power solutions for use in industry predominantly use electromechanical and electronic meters to measure, display and record consumption locally. The Eco-eye [46] system consists of nodes that use CT clips to monitor and measure three-phase power, transmitting data to a remote screen. Eco-eye nodes can be calibrated to match specific loads between $90-255 \mathrm{~V}$ and measure up to $200 \mathrm{~A}$ per phase, but are not connected to the Internet and cannot provide historical data. A remote display solution developed by Efergy [47] used nodes to measure voltage ranges of $110-300 \mathrm{~V}$ and current between 50mA-95A. Efergy nodes maintain local historical data but cannot display updates through the Internet or provide process-level usage analysis.

Siemens [48] provides the 7KM series of products, capable of monitoring high power machines at high frequency. The meters are designed for factory installation on DIN rails and can accurately measure active and reactive energy. The system does offer database storage of measurements that can be accessed through local stations or over the Internet. Additional Siemens software can then provide historical and real-time power monitoring, as well as power-management capabilities. However, in supporting Ethernet but not wireless communications, implementation requires the addition and maintenance of an intrusive infrastructure of cables.

Guimaraes et al [12] propose a three-phase energy monitor, with additional power factor, overvoltage and overcurrent monitoring. The system uses an ADE7758 IC to measure active, reactive and apparent power and real and apparent energy. Non-invasive sensing is performed using CTs, to monitor current up to a maximum of 10A and a voltage transformer capable of monitoring up to 220V. Data are transferred to an ATmega microcontroller via an SPI bus, and output to LCD display.

Schneider Electric offers an energy and power management system, called Powerlogic, to support industry, 

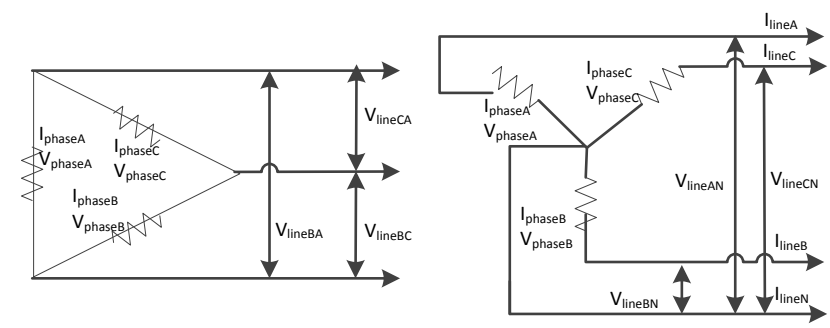

Figure 21: Phase and Line V and I in Wye and Delta

building, critical infrastructure and utilities' monitoring. A range of power meters, communication hardware and monitoring software are implemented for real-time monitoring of up to $600 \mathrm{~V} \mathrm{AC}$, with remote access [49]. However, prediction and optimisation of power consumption are not offered.

A cyber physical system monitors, coordinates, controls and integrates the operations of an engineered physical system, using a computing and communication system, to improve the efficiency offered by the physical system alone [50]. Monitoring and prediction of process energy consumption is thus complemented by automated, efficient control of consumption. This can be optimised according to pre-agreed billing thresholds, resource availability and key performance indicators such as production yield, defect rate and equipment efficiency.

The WEMS (Wireless Energy Management Systems International Ltd) system offers a wireless solution for energy management within buildings, metering total energy usage for a site and environmental conditions to control equipment on site. The system provides a range of services to remotely monitor and manage energy usage across multiple sites, but lacks the means to identify machine level power signatures [51]. Energy measurement systems for high-power, three-phase industrial systems have not taken a cyber physical IoT approach. A requirement still exists for low-power, remotely accessible and reconfigurable, scalable energy monitoring of active, reactive and apparent power in industrial (> 400 V) systems and environments and for optimisation of these systems to reduce waste.

\subsection{Three-phase Calculation}

Three-phase Wye and Delta configurations are used to supply high power loads. Delta is commonly used for industrial loads, as different voltage combinations can be obtained by making connections along the windings of supply transformers and high power can be supplied to a load. Wye and Delta require four (phase AB-C and Neutral for unbalanced loads) or three wires (phase A-B-C for balanced loads). In the former, one

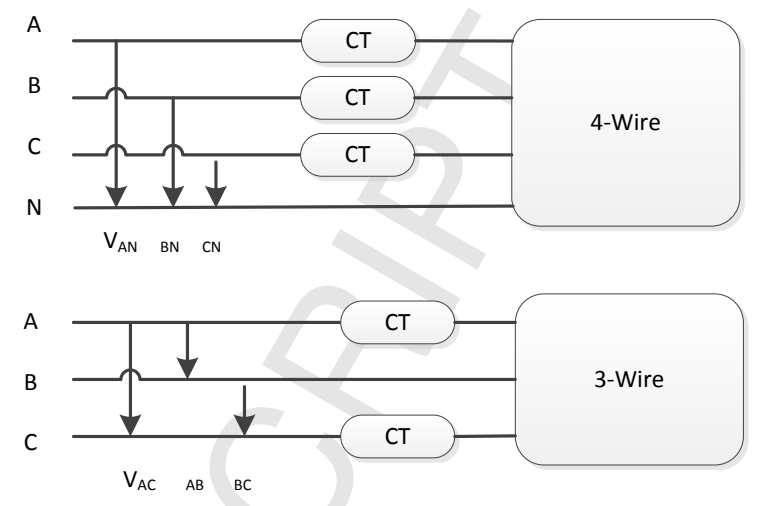

Figure 22: Systems With and Without Neutral

phase is used as the reference point for the distribution. The Cyber-Physical Toolset in this paper is suitable for three and four-wire systems, with devices configured remotely to identify the wiring configuration monitored.

Voltages and current across generator windings are called phase voltage $\left(V_{\text {phase }}\right)$ and phase current $\left(I_{\text {phase }}\right)$. Voltages and currents measured across $(\mathrm{V})$ or through (I) any two lines connecting generator to load are called line voltage $\left(V_{\text {line }}\right)$ and line current $\left(I_{\text {line }}\right)$ [52] (see Figure 21). In Wye configurations the line and phase currents are equal $\left(I_{\text {phase }}=I_{\text {line }}\right)$ and $V_{\text {line }}=\sqrt{3} V_{\text {phase }}$. In Delta configurations the line and phase voltages are equal $\left(V_{\text {phase }}=V_{\text {line }}\right)$ and $I_{\text {line }}=\sqrt{3} I_{\text {phase }}$.

Where $V_{k}$ and $I_{k}$ are RMS measurements of sinusoidal voltage and current variation, equation 1 demonstrates the calculation of the supplied voltage, $v(t)$ and consumed current, $i(t)$, when $\phi_{k}$ and $\gamma_{k}$ are phase delays of each waveform. Instantaneous power for individual phases can be calculated from $p(t)=v(t) \cdot i(t)$.

$$
\begin{gathered}
v(t)=\sum_{k=1}^{\infty} V_{k} \sqrt{2} \sin \left(k \omega t+\phi_{k}\right) \\
i(t)=\sum_{k=1}^{\infty} I_{k} \sqrt{2} \sin \left(k \omega t+\gamma_{k}\right) \\
F_{k}=\sqrt{\sum_{k=0}^{N-1} \frac{F^{2} k}{N}}
\end{gathered}
$$

$$
P_{\text {active }}=\frac{1}{n T} \int_{0}^{n T} p(t) d t=\sum_{k=1}^{\infty} V_{k} I_{k} \cos \left(\phi_{k}-\gamma_{k}\right)
$$

The discrete time equations for the calculation of $V_{k}$ and $I_{k}$ use equation 2 , where $N$ is the number of samples and $F$ is the line measurement [11]. Total active power 
is then calculated by the expression in equation 3 , where $T$ is the line cycle period and $n$ is the number of cycles over which the average is to be calculated.

Reactive and apparent power are calculated using $P_{\text {apparent }}=V_{k} \times I_{k}$ and $P_{\text {reactive }}=\sqrt{P_{\text {apparent }}^{2}-P_{\text {active }}^{2}}$. Figure 22 demonstrates voltage and current measurement in three and four-wire three-phase configurations. For three wire systems, two line current $\left(I_{A}, I_{C}\right)$ and voltage readings $\left(V_{A B}, V_{C B}\right)$ suffice, as the potential of line B can be used as the reference point [53]. Fourwire configurations require three current $\left(I_{A}, I_{B}, I_{C}\right)$ and three voltage readings between phase and neutral (VAN, $\left.V B N, V_{C N}\right)$.

\subsection{Energy Consumption Prediction}

A method for predicting the energy consumption of the main driving system of a machine tool was proposed by [54], by dividing the machining process into start-up, idle and cutting periods and establishing energy consumption prediction models for each process. Similarly, [55] investigated the correlation between numerical control (NC) codes and the energy-consuming components of machine tools. They proposed a method for estimating the energy consumption of NC machining by estimating the contribution of each component.

Various simulations for the prediction of energy consumption have been based on frictional energy loss modelling. The authors in [56] predicted motor current using a component-based simulation model. To enhance the model accuracy, a friction model including time-dependent frictional characteristics and rolling contact conditions was introduced. A model to predict the power consumption of ball screw and linear motor feed drive systems has also been proposed [57].

The model was able to accurately predict the power consumption of the ball screw feed drive system, but not the linear motor. The authors in [58] use the MATLAB SimEvents toolbox to predict consumption on the basis of CNC G-code for a turning operation, assuming that each tool component exists only in 'on' or 'off' states, with constant consumption when 'on'. The stiffness and frequency response of machine tool feed drives have also been predicted through simulation $[59,60]$.

The CNC machine, monitored in this paper used a three-phase induction (spindle) motor, which has been modelled in MATLAB Simulink, as described in Section 2.3. A generalised model of a three-phase induction motor has been developed using Simulink [61]. Other models have also been developed to simulate Direct Self Control [62] and Direct Torque Control Drives [63].
The Simulink SimPowerSystems software provides component libraries and analysis tools for modelling and simulating electrical power systems, such as the SimPowerSystems Library, a Three-Phase, Stair Generator, speed reference and torque and Power blocks. These have been used in this paper to model an AC drive to more accurately predict the energy usage of a CNC spindle motor (as described in Section 2.3). The three-phase voltages were generated such that $V_{a s}=$ $|V| \cos (\omega t+\phi), V_{b s}=|V| \cos (\omega t-2 \pi / 3+\phi)$ and $V_{c s}=$ $|V| \cos (\omega t+2 \pi / 3+\phi)$. Where Where $|V|$ is the terminal voltage amplitude, $\omega$ is the angular frequency of the supply, and $\phi$ is the initial phase angle.

\section{Conclusions and Future Work}

This paper has presented a novel IoT industrial energy monitoring Cyber-Physical Toolset that consists of four layers: (1) The smart factory-floor machineto-sensor interaction, providing robust, high-accuracy metering (2) The WSN and LAN infrastructure which allows secure data-transfer for distributed systems, incorporating the machines into the IoT (3) The remote information repository which provides monitoring and data analysis services (4) The client layer with an efficient control service to ensure the best scheduling and production strategy that also falls within limits set by policy makers and energy providers and power prediction, reducing the necessity for monitoring to intrude on supply chain infrastructure.

The Cyber-Physical Toolset has been evaluated both with a three-phase industrial emulator system and monitoring a Hurco VM1 CNC Machine. Validation demonstrates that an IoT approach can support intelligent monitoring of process power cycles and tuning of threephase energy usage in high-power industrial environments. This can then be used to achieve resource efficiency, minimise production costs and cut $\mathrm{CO}_{2}$ emissions for a greener industrial entity.

As future work the Cyber-Physical Toolset will be applied to a wider range of manufacturing domains, commencing with Laser Micro Assembly and PCB assembly line machines. The efficient control service will be further enriched with abstracted knowledge of the UK National Grid real-time energy pricing information.

\section{References}

[1] U.S Energy Information Administration, International Energy Outlook 2016, Washington, DC, USA, 2016, vol. 0484, no. May. 
[2] D. of Energy and C. Change, "2014 UK Greenhouse Gas Emissions, Provisional Figures and 2012 UK Greenhouse Gas Emissions , Final Figures by Fuel Type and End-User Statistical Release," p. 44, Mar. 2014.

[3] F. Li, J. W. Marangon-Lima, Hugh Rudnick, L. M. MarangonLima, N. P. Padhy, G. Brunekreeft, J. Reneses, and C. Kang, "Distribution Pricing," IEEE Power and Energy Magazine, pp. 76-86, Aug. 2015.

[4] S. Thiede, Energy Efficiency in Manufacturing Systems. Springer Publishing Company, Incorporated, 2012.

[5] European Commission. (2013, Dec.) Europe 2020 Targets: Climate Change and Energy.

[6] McKinsey \& Company, "Unlocking Energy Efficiency in the U.S. Economy," Jul. 2009.

[7] CBI. (2013) Government must untangle overlapping policies that deter energy efficiency investments.

[8] U.S. DOE. (2003) Energy Information Administration, Commercial Buildings Energy Consumption Survey.

[9] A. Vijayaraghavan and D. Dornfeld, "Automated Energy Monitoring of Machine Tools," CIRP Annals - Manufacturing Technology, vol. 59, no. 1, pp. 21-24, 2010.

[10] IEC, "Low-voltage Switchgear and Controlgear Assemblies," IEC, Tech. Rep., 2011.

[11] (2016, Apr.) How to Build an OpenEnergyMonitor system.

[12] A. Guimaraes, T. Freitas, H. Griner, and T. de Almeida, "Smart Energy Monitoring System with ADE7758 IC," in Energy, 5th International Youth Conference on, May 2015, pp. 1-5.

[13] J. F. Whitfield and A. Hay-Ellis, The Electricians Guide to the 17th Ed. of the IEE Wiring Regulations BS 7671: 2011 and Part $P$ of the Building Regulations. EPA Press, 2015.

[14] DCUSA, "Distribution Connection and Use of System Agreement," Office of Gas and Electricity Markets (Ofgem), Tech Rep., Oct. 2006.

[15] Department of Energy and Climate Change, "Digest of United Kingdom Energy Statistics," Tech. Rep., 2014

[16] — " "Quarterly energy prices, December," Tech. Rep., 2014.

[17] K. Vikhorev, R. Greenough, and N. Brown, "An Advanced Energy Management Framework to Promote Energy Awareness," Journal of Cleaner Production, 2013.

[18] J. Lifton, M. Feldmeier, Y. Ono, C. Lewis, and J. a. Paradiso, "A Platform for Ubiquitous Sensor Deployment in Occupational and Domestic Environments," Proc. of the 6th International Conference on Information Processing in Sensor Networks (IPSN '07), pp. 119-127, 2007.

[19] Npower, "Small and Medium-sized Enterprises Deemed Prices," Tech. Rep., Apr. 2015.

[20] Ford Motor Company, "Ford Renewable Energy Sustainability Report 2013/14," Tech. Rep., 2014.

[21] D. Gordić, M. Babić, N. Jovičić, V. Šušteršič, D. Končalović, and D. Jelić, "Development of Energy Management System Case study of Serbian Car Manufacturer," Energy Conversion and Management, vol. 51, no. 12, pp. 2783-2790, 2010.

[22] Ford Motor Company, "Ford Continues High-tech Engine Investment at Dagenham," 2014

[23] W. Shen and D. H. Norrie, "Agent-Based Systems for Intelligent Manufacturing : A State-of-the-Art Survey," Knowledge and Information Systems, vol. 1, no. 2, pp. 129-156, Mar. 1999.

[24] D. Miorandi, S. Sicari, F. De Pellegrini, and I. Chlamtac, "Internet of Things: Vision, Applications and Research Challenges," Ad Hoc Networks, vol. 10, no. 7, pp. 1497-1516, 2012

[25] L. Xu, W. He, and S. Li, "Internet of Things in Industries: A Survey," IEEE Transactions on Industrial Informatics, vol. PP, no. 4, pp. 1-11, 2014

[26] Texas Instruments, “Application Notes cc2538+cc1200," Tech. Rep., Sep. 2013.
[27] ARM, "Cortex-M4 Processor," ARM, Tech. Rep.

[28] I. Akyildiz, W. Su, Y. Sankarasubramaniam, and E. Cayirci, "Wireless Sensor Networks: a Survey," Computer Networks, vol. 38, no. 4, pp. 393-422, 2002

[29] T. Winter, P. Thubert, B. Anders, J. Hui, R. Kelsey, P. Levis, K. Pister, R. Struik, J. P. Vasseur, and R. Alexander, "RPL: IPv6 Routing Protocol for Low power and Lossy Networks,' RFC 6550, Mar. 2012.

[30] Jonathan Hui and J. P. Vasseur and Cisco Systems, "The Routing Protocol for Low-Power and Lossy Networks (RPL) Option for Carrying RPL Information in Data-Plane Datagrams," RFC 6553, Mar. 2012.

[31] “About Contiki," Feb. 2010.

[32] Z. Shelby, K. Hartke, and C. Bormann, "Constrained Application Protocol (CoAP)," IETF, Tech. Rep., Jun. 2013.

[33] Hurco, "VM1 and VMX1 Machining Centers Technical Specification," Tech. Rep., 2005.

[34] I. Engineering and A. M. Performance, "Key Performance Indicators for Sustainable Manufacturing Evaluation in Automotive Companies," pp. 1093-1097, 2011.

[35] H. Nguyen, "Manufacturing Processes and Engineering Materials Used in Automotive Engine Block," Ph.D. dissertation, Grand Valley State University, 2005.

[36] Gibbs Machinery, "4.0 Litre Cylinder Block Machining Line." [Online]. Available: http://www.gibbsmachinery.com/ email/40cylblock.html

[37] AB Sandvik Coromant, "Efficient machining of Crankshafts and Camshafts,” Düsseldorf, Tech. Rep., 2003.

[38] Ford Motor Company, "Ford Celebrates Production of 40 million Engines at Dagenham," [Online; accessed 25-July-2016].

[39] “GDF Suez Drives Energy Management at Ford," 2011.

[40] D. of Energy and C. Change, "Industrial Electricity Prices in the IEA (QEP 5.3.1) Statistical Data Set," Tech. Rep., Oct. 2015.

[41] _ "Quarterly: Industrial Electricity Prices in the EU for Small, Medium, Large and Extra Large Consumers Statistical Data Set," Tech. Rep., Oct 2015.

[42] A. Ruzzelli, C. Nicolas, A. Schoofs, and G. M. P. O'Hare, "Real-Time Recognition and Profiling of Appliances Through a Single Electricity Sensor," in Sensor Mesh and Ad Hoc Communications and Networks (SECON), 2010 7th Annual IEEE Communications Society Conference on, Jun. 2010, pp. 1-9.

[43] G. Bauer, K. Stockinger, and P. Lukowicz, "Recognizing the Use-Mode of Kitchen Appliances from Their Current Consumption," in Proceedings of the 4th European Conference on Smart Sensing and Context. Berlin, Heidelberg: Springer-Verlag, 2009, pp. 163-176.

[44] S. O'Connell, J. Barton, E. O’Connell, B. O'Flynn, E. Popovici, S. O'Mathuna, A. Schoofs, A. Ruzzelli, and G. O'Hare, "Remote Electricity Actuation and Monitoring Mote," in Distributed Computing in Sensor Systems and Workshops (DCOSS), International Conference on, Jun. 2011, pp. 1-6.

[45] X. Jiang, S. Dawson-Haggerty, P. Dutta, and D. Culler, "Design and Implementation of a High-fidelity AC Metering Network," in Proceedings of the 2009 International Conference on Information Processing in Sensor Networks, ser. IPSN '09. Washington, DC, USA: IEEE Computer Society, 2009, pp. 253-264.

[46] Eco-Eye. (2016, May) ELITE200 Three Phase Energy Monitor.

[47] Efergy. (2016, Apr.) Three-phase Elite Monitor.

[48] S. AG. (2013) Measuring Devices and Power Monitoring.

[49] Square D, "Ford Motor Company Implements Schneider Electrics Enterprise Energy Management System," Schneider Electric, Tech. Rep., 2010.

[50] R. Rajkumar, I. L. I. Lee, L. S. L. Sha, and J. Stankovic, "Cyberphysical systems: The next computing revolution," Design Automation Conference (DAC), 47th ACM/IEEE, pp. 0-5, 2010. 
[51] Wireless Energy Management Systems International (WEMS), "Energy Services Brochure," WEMS International The Mission, Wellington Street, Stockport, Greater Manchester, SK1 3AH, United Kingdom, Tech. Rep.

[52] T. L. Floyd, Principles of Electric Circuits. Prentice Hall, 2003.

[53] Tektronix, "The Fundamentals of Three-Phase Power Measurements," Tech. Rep., Jun. 2013.

[54] F. Liu, J. Xie, and S. Liu, "A method for predicting the energy consumption of the main driving system of a machine tool in a machining process," Journal of Cleaner Production, vol. 105, pp. 171-177, 2015.

[55] Y. He, F. Liu, T. Wu, F.-P. Zhong, and B. Peng, "Analysis and estimation of energy consumption for numerical control machining," Proceedings of the Institution of Mechanical Engineers, Part B: Journal of Engineering Manufacture, vol. 226, no. 2 , pp. 255-266, 2011.

[56] Y. H. Jeong, B. K. Min, D. W. Cho, and S. J. Lee, "Motor current prediction of a machine tool feed drive using a component-based simulation model," International Journal of Precision Engineering and Manufacturing, vol. 11, no. 4, pp. 597-606, 2010.

[57] A. HAYASHI, R. SATO, and K. SHIRASE, "Measurement and Evaluation of Electric Power Consumption of Feed Drive Systems in NC Machine Tool," Journal of the Japan Society for Precision Engineering, vol. 79, no. 10, pp. 930-936, 2013.

[58] R. Larek, E. Brinksmeier, D. Meyer, T. Pawletta, and O. Hagendorf, "A discrete-event simulation approach to predict power consumption in machining processes," Production Engineering, vol. 5, no. 5, pp. 575-579, 2011.

[59] M. Ebrahimi and R. Whalley, "Analysis, Modeling and Simulation of Stiffness in Machine Tool Drives," Computers and In dustrial Engineering, vol. 38, no. 1, pp. 93-105, 2000.

[60] S. Frey, a. Dadalau, and a. Verl, "Expedient Modeling of Ball Screw Feed Drives," Production Engineering, vol. 6, no. 2, pp. 205-211, 2012.

[61] K. L. Shi, T. F. Chan, Y. K. Wong, and S. L. Ho, "Modelling and Simulation of the Three-phase Induction Motor Using Simulink," International Journal of Electrical Engineering Education, vol. 36, pp. 163-172, 1999.

[62] K. L. Shi, T. F. Chan, Y. KWong, S. L. Ho, "Modelling and Simulation of Direct Self-Control Systems," The International Journal of Engineering Education, vol. 19, no. 4, pp. 646-654, 2003.

[63] Z. a. Alnasir and a. H. Almarhoon, "Design of Direct Torque Controller of Induction Motor,' International Journal of Engineering and Technology, vol. 4, no. 2, pp. 54-70, 2012

[64] Lanner Group, Learning WITNESS: Book One, Manufacturing Performance Edition, 2013.

[65] Lanner Group, Learning WITNESS: Book Two - Workbook Manufacturing Performance Edition, 2013.

[66] Lanner Group Limited, "Getting Started How to install Witness and start using it," Tech. Rep., 2008.

[67] A. Waller, "WITNESS Simulation Software," in Proceedings of the 2012 Winter Simulation Conference, C. Laroque, J. Himmelspach, R. Pasupathy, O. Rose, and A. Uhrmacher, Eds., 2012, pp. 1-12. 


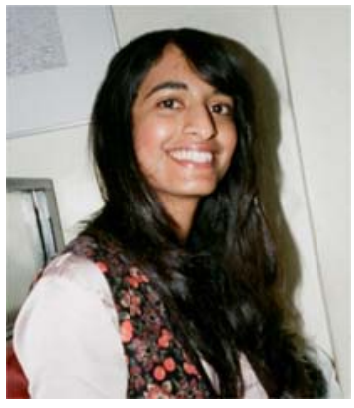

Sarogini Grace Pease is a Research Associate at the Wolfson School of Mechanical, Electrical and Manufacturing Engineering at Loughborough University. She holds a Ph.D. degree, funded under CASE award from the Engineering and Physical Sciences Research Council and B.A.E. Systems, and an M.Sc. degree from the Department of Computer Science, Loughborough University. Dr Pease also holds an LL.B.(Hons.) degree in law from the University of Essex. She previously held Communications Manager positions at H.M. Prison Service, Dresdner Kleinwort Investment Bank and The London Museums Hub. Her research interests are in the design and performance improvement of real-time mobile ad hoc and wireless sensor networks and intelligent industrial monitoring and tracking systems and networks.

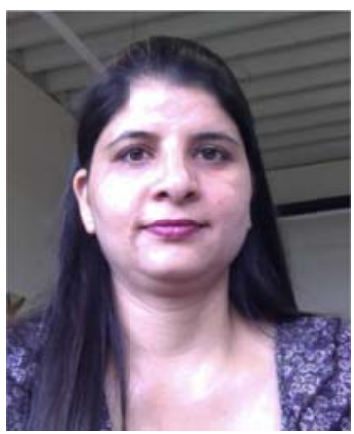

Navjot Kaur received her B.E. degree in Electronics \& Communication Engineering from GZCET, Bathinda, India in 2000 and M.Tech. in Computer Science Engineering from Punjabi University, Patiala, India in 2003. She obtained a Ph.D. degree in Mechanical \& Manufacturing Engineering from Loughborough University, UK in 2012 and continued working as Research Associate in the same department till 2016. Her main research has focused on the implementation of Service Oriented Architectures and component based manufacturing automation in power train engine assembly lines and the design of UHF RFID tags in harsh environments for tracking and supply chain monitoring.

Russell Trueman is a graduate student of Loughborough University, Wolfson School of Mechanical, Electrical and Manufacturing Engineering he received his master degree in mechanical engineering in 2015. 


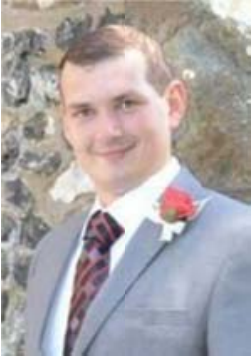

Callum Davies is a graduate student of Loughborough University, Wolfson School of Mechanical, Electrical and Manufacturing Engineering he received his master degree in mechanical engineering in 2015.

Jude Grosberg is a graduate student of Loughborough University, Wolfson School of Mechanical, Electrical and Manufacturing Engineering he received his master degree in mechanical engineering in 2015.

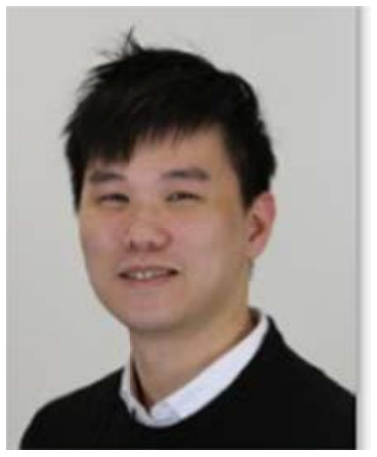

Kai Hin Yau is a graduate student of Loughborough University, Wolfson School of Mechanical, Electrical and Manufacturing Engineering he received his master degree in mechanical engineering in 2015. He is currently working in an E\&M consultancy of the construction industry.

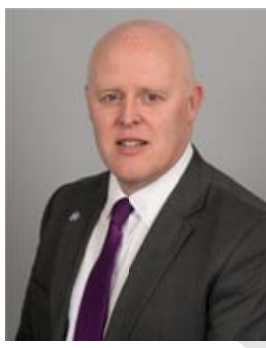

Paul P. Conway is the Professor of Manufacturing Processes in the Wolfson School of Mechanical, Electrical and Manufacturing Engineering at Loughborough University. He holds degrees from the University of Ulster and Loughborough University. He led the Research Council UK's (RCUK) Innovative Electronics Manufacturing Research Centre from 2004 for 10 years. This Centre became the beacon for electronics manufacturing research in Great Britain and an internationally recognised Centre of excellence in that field. He holds several executive and non-executive positions such as the 
Director of the RCUK's Centre for Doctoral Training in Embedded Intelligence. His research interests are in the areas of manufacturing intelligence, embedded sensors and multifunctional materials manufacturing. He is a senior member of IEEE and Fellow of the Institution of Mechanical Engineers.

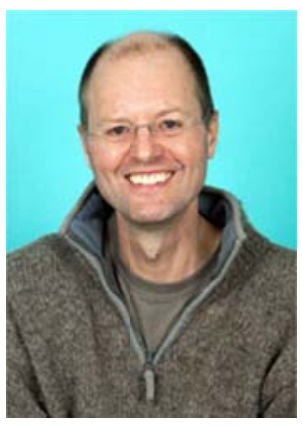

Andrew A. West is currently a Professor of intelligent systems with the Wolfson School of Mechanical, Electrical and Manufacturing Engineering, Loughborough University, Loughborough. He has over 25 years of experience in research and industrial consultancy with the University of Leeds, Leeds; with Cambridge University, Cambridge. and with Loughborough University. He is the author of over 170 journal and conference research publications. His collaborative research with the Engineering and Physical Sciences Research Council, the European Union, and the U.K. Technology Strategy Board has generated over \$25 million income to Loughborough University. His main research interests include lifecycle engineering of intelligent distributed component-based manufacturing control and monitoring systems. 
- A novel real-time power and process prediction and optimisation solution is proposed in this paper.

- A high power machine energy monitoring architecture is extended to a cyber-physical toolset using a Wireless Sensor Network (WSN) of high accuracy, low-power Dynamic Resource Monitors (DRM) and remote servers, for real-time energy signature characterisation services as well as user access via databases and web interfaces.

- An energy and power consumption prediction service is developed to generate a precise visualisation of expected power usage on the basis of measurements of real automotive industry operations and processes.

- An efficient control service is shown to respond to time-varying energy requirements to bound total plant energy consumption to variable thresholds.

- Extensive validation of the prediction capabilities of the architecture is conducted against real measurements from an automotive production line to ensure that total plant consumption and productivity remain within acceptable and guaranteed bounds to avoid peak charges and reduce waste. 